\title{
Dynamic recontracting processes with multiple indivisible goods
}

Citation for published version (APA):

Bochet, O. L. A., Klaus, B. E., \& WalzI, M. (2007). Dynamic recontracting processes with multiple indivisible goods. METEOR, Maastricht University School of Business and Economics. METEOR Research Memorandum No. 018 https://doi.org/10.26481/umamet.2007018

Document status and date:

Published: 01/01/2007

DOI:

10.26481/umamet.2007018

Document Version:

Publisher's PDF, also known as Version of record

\section{Please check the document version of this publication:}

- A submitted manuscript is the version of the article upon submission and before peer-review. There can be important differences between the submitted version and the official published version of record.

People interested in the research are advised to contact the author for the final version of the publication, or visit the DOI to the publisher's website.

- The final author version and the galley proof are versions of the publication after peer review.

- The final published version features the final layout of the paper including the volume, issue and page numbers.

Link to publication

\footnotetext{
General rights rights.

- You may freely distribute the URL identifying the publication in the public portal. please follow below link for the End User Agreement:

www.umlib.nl/taverne-license

Take down policy

If you believe that this document breaches copyright please contact us at:

repository@maastrichtuniversity.nl

providing details and we will investigate your claim.
}

Copyright and moral rights for the publications made accessible in the public portal are retained by the authors and/or other copyright owners and it is a condition of accessing publications that users recognise and abide by the legal requirements associated with these

- Users may download and print one copy of any publication from the public portal for the purpose of private study or research.

- You may not further distribute the material or use it for any profit-making activity or commercial gain

If the publication is distributed under the terms of Article $25 \mathrm{fa}$ of the Dutch Copyright Act, indicated by the "Taverne" license above, 
Olivier Bochet, Bettina Klaus, Markus Walzl

Dynamic Recontracting Processes with Multiple Indivisible Goods

$\mathrm{RM} / 07 / 018$

JEL code: D63, D70

\section{METEबR}

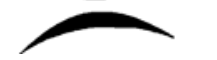

Maastricht research school of Economics

of TEchnology and ORganizations

Universiteit Maastricht

Faculty of Economics and Business Administration

P.O. Box 616

NL - 6200 MD Maastricht

phone : ++31433883830

fax : :+31433884873 



\title{
Dynamic Recontracting Processes with Multiple Indivisible Goods*
}

\author{
Olivier Bochet $\dagger$ Bettina Klaus; and Markus Walzl ${ }^{\S}$ \\ Maastricht University, Department of Economics
}

May 2007

\begin{abstract}
We consider multiple-type housing markets. To capture the dynamic aspect of trade in such markets, we study a dynamic recontracting process similar to the one introduced by Serrano and Volij (2005). First, we analyze the set of recurrent classes of this process as a (non-empty) solution concept. We show that each core allocation always constitutes a singleton recurrent class and provide examples of non-singleton recurrent classes consisting of blocking-cycles of individually rational allocations. For multiple-type housing markets stochastic stability never serves as a selection device among recurrent classes.

Next, we propose a method to compute the limit invariant distribution of the dynamic recontracting process. The limit invariant distribution exploits the interplay of coalitional stability and accessibility that determines a probability distribution over final allocations. We provide various examples to demonstrate how the limit invariant distribution discriminates among stochastically stable allocations: surprisingly, some core allocations are less likely to be final allocations of the dynamic process than cycles composed of non-core allocations.

Keywords: core, indivisible goods, limit invariant distribution, stochastic stability. JEL classification: D63, D70.
\end{abstract}

\section{Introduction}

Dynamic recontracting processes Consider Shapley and Scarf's (1974) well-known model of exchange with indivisible goods, the so-called housing market model. ${ }^{1}$ One of the most important solution concepts for such markets is the core. An allocation $x$ is in the core if there does not exist a coalition that can improve upon $x$ using its own endowments: $x$ cannot be blocked. ${ }^{2}$ The core of a housing market satisfies some remarkable properties. Most prominently, the core consists of a unique allocation that also turns out to be the unique Walrasian allocation of the market (Roth and Postlewaite, 1977). Furthermore, the core is a

\footnotetext{
${ }^{*}$ We are grateful to Juan D. Moreno-Ternero and William Thomson for their useful comments. B. Klaus thanks the Netherlands Organisation for Scientific Research NWO for its support under grant VIDI-452-06-013.

${ }^{\dagger}$ e-mail: o.bochet@algec.unimaas.nl

${ }^{\ddagger}$ Corresponding author: Department of Economics, Maastricht University, P.O. Box 616, 6200 MD Maastricht, The Netherlands; e-mail: b.klaus@algec.unimaas.nl

$\S$ e-mail: m.walzl@algec.unimaas.nl

${ }^{1}$ In Shapley-Scarf housing markets, each agent is endowed with a house, has strict preferences over the set of houses in the market, and wishes to consume exactly one house.

${ }^{2}$ Note that the core we introduce here is sometimes referred to as the strong (or strict) core. The notion of blocking associated with the strong core is weak blocking. That is, when a coalition $S$ blocks $x$, no member of $S$ is worse-off and at least one of its member is better-off.
} 
static solution concept "with a dynamic heart": it is defined for a fixed economy, but if agents block, they cause a transition from one state of the world (an allocation) to another state of the world (an allocation where the members of the blocking coalition are better off), which hints at a dynamic process that however is not explicitly modeled. So the core incorporates robustness against potential transitions in a model that does not accommodate the possibility of transition.

Our aim here is to better understand the dynamics of trade. In particular we are interested in its resulting allocations - inside as well as outside the core. To this end, we study the following dynamic recontracting process or d.r. process for short. The d.r. process starts with the agents' endowments as the initial allocation for trade. Throughout the d.r. process we do not redefine endowments (property rights are not exchanged). At any stage agents can recontract upon the allocation $x$ that resulted from previous trades. A coalition is randomly selected and is allowed to recontract over $x$ if it can block $x$ using its endowments. A new allocation is obtained as follows: agents in the coalition reallocate their endowments according to the blocking. If this reallocation is feasible because no agent outside the coalition was consuming the endowment of an agent in the coalition, then agents outside the coalition stick to their assignment at $x$. If the coalition's recontracting is not feasible, then agents outside the coalition receive their endowments. Thus, at each period, a coalition is randomly selected and has the power to make the process transit from the prevailing allocation to another one. This determines a Markov process on the set of allocations. ${ }^{3}$ In the long-run, such a Markovprocess always ends up in one of its recurrent classes: a set of allocations that once reached will never be abandoned.

To select among recurrent classes, we allow that agents make mistakes when they recontract; we "perturb" the d.r. process. This means that in every period, each agent with a small probability $\epsilon$ agrees on a reallocation that makes him worse off. In such a perturbed d.r. process, any allocation can be reached from any other allocation after a finite number of periods (with sufficiently many mistakes by the agents involved). Hence, a perturbed d.r. process has only one recurrent class - the entire set of allocations - and the probability distribution over allocations induced by the perturbed d.r. process converges (in the long-run and for small $\epsilon$ ) to the so-called limit invariant distribution, which is unique. The support of this distribution - the set of stochastically stable allocations - is the set of allocations to which the perturbed d.r. process converges with strictly positive probability. Hence, stochastically stable allocations can be regarded as the (potentially) final allocations of the d.r. process, and the limit invariant distribution is a probability distribution over these candidate final allocations.

Note that similarly as the core, recurrent classes of the unperturbed d.r. process are static. A set of allocations is a recurrent class if it exhibits the following stability: no allocation outside the recurrent class blocks an allocation in the recurrent class. In contrast, the limit invariant distribution is an inherently dynamic concept as it also captures the accessibility of an allocation. The easier it is for the d.r. process to reach an allocation - and, the more difficult to exit - the larger is the probability that the d.r. process converges to the respective allocation in the long run. Hence, the limit invariant distribution is a probability distribution over final allocations that combines a core-like stability concept (each stochastically stable allocation is an element of a recurrent class) with a notion of accessibility incorporated by the d.r. process.

\footnotetext{
${ }^{3}$ We discuss basic notions and terminology of Markov processes in Appendix B.
} 
One of our objectives is a computational method that elicits the set of stochastically stable allocations and the limit invariant distribution. In particular, we are interested in the relation between the set of final allocations of d.r. processes (and the respective probability distributions) and the core. This is the main reason why we have modeled the d.r. process using recontracting based on core blocking and without the transfer of property rights (once property rights are transferred throughout the process, it is obvious that the core of the initial market will not play any specific role). Given that we model the d.r. process as closely as possible to the implicit dynamic elements incorporated into the core, we ask the following questions: Are core allocations necessarily elements of recurrent classes and stochastically stable? Does the process converge to every core allocation with the same probability? Can the process converge to non-core allocations? And, what is the relation of all these solution concepts to Walrasian allocation(s)?

Relation to the Literature Pioneering work on d.r. processes for exchange economies has been conducted by Feldman (1974) and Green (1974). They provide conditions for which a d.r. process converges to the core and thereby formalize Edgeworth's intuition that the final allocation of an exchange economy can be reached through dynamic recontracting. In a recent contribution, Serrano and Volij (2005) use d.r. processes to analyze Shapley-Scarf housing markets. Using a Markov process identical to the one described above, they show that the unique core allocation is the unique recurrent class (and, hence, the unique stochastically stable allocation) of the d.r. process. This "equivalence" result between the core and the set of final allocations of the d.r. process is driven by the global dominance property of the core for Shapley-Scarf housing markets. ${ }^{4}$ Next, Serrano and Volij (2005) extend the classical housing market model by allowing for indifferences in the agents' preferences. For this model, they characterize the set of recurrent classes and stochastically stable allocations. In particular, they show that every allocation in the core forms a singleton recurrent class of the d.r. process. However, not every core allocation is stochastically stable. They provide examples where (i) the set of stochastically stable allocations coincides with the set of core allocations, (ii) requiring stochastic stability selects certain core allocations that are not necessarily Walrasian, and (iii) the set of stochastically stable allocations overlaps with the set of core allocations but also contains cycles of non-core allocations. These results suggest that the set of stochastically stable allocations is a dynamic solution concept that relates to the core in a non-trivial way. In particular, they demonstrate how stochastic stability serves as a selection device among recurrent classes.

Our Contribution We consider a different extension of Shapley-Scarf housing markets than Serrano and Volij (2005). We keep preferences strict, but extend the analysis to multipletype housing markets (Moulin, 1995). Hence, we endow each agent with one commodity of each type (e.g., houses and cars or, more realistically, tutor groups for different courses as described in Klaus, 2006) and analyze simultaneous trade in all these types. Konishi, Quint, and Wako (2001) show that the core of such an economy may well be empty or multi-valued. ${ }^{5}$ For the case of only one type our model is identical to Serrano and Volij's (2005) benchmark model with strict preferences.

\footnotetext{
${ }^{4}$ Roth and Postlewaite (1977) demonstrate that in a Shapley-Scarf housing market each allocation outside the core can be blocked by the unique core allocation.

${ }^{5}$ We show that the same holds for the set of Walrasian allocations.
} 
Similar to Serrano and Volij (2005), we show that each allocation in the core forms a singleton recurrent classes of the d.r. process while there are possibly non-singleton recurrent classes consisting of blocking cycles. In contrast to Serrano and Volij's findings - and to many applications of Markov processes (see for example the literature on equilibrium selection in non-cooperative games as proposed by Kandori et al., 1993; Young, 1993) - we show that stochastic stability never serves as a selection device among recurrent classes. There is no recurrent class of the d.r. process that fails to be stochastically stable. However, this does not imply that each stochastically stable allocation (each allocation in the support of the limit invariant distribution) will be the final allocation of the process with the same probability.

Starting with a result by Freidlin and Wentzell (1998), we develop a method to compute the limit invariant distribution of a d.r. process. As the limit invariant distribution is a probability distribution over stochastically stable recurrent classes, its use is to discriminate between the different stochastically stable allocations as contenders for the final allocations of the economy. This discrimination hinges on one crucial conceptual difference between what determines the stochastic stability of an allocation and what determines the probability that this allocation will be the final allocation of the process. While stochastic stability solely depends on the minimum number of mistakes needed to reach and leave a certain allocation, the probability assigned by the limit invariant distribution is also determined by the transition probabilities of the original d.r. process. This additional dependence on the underlying d.r. process allows for a finer characterization of its final allocations.

We illustrate the computational techniques and their interpretation with several examples. In particular we show that some core and Walrasian allocations may be the least likely of all possible final allocations. On the other hand, blocking-cycles may emerge as powerful contenders for final allocations even if the core is non-empty. Hence, following the long-run predictions of a d.r. process leads us to a better understanding of the non-trivial relation between core allocations, Walrasian allocations, and blocking-cycles.

Moreover, we regard our analysis of d.r. processes as an instructive illustration of a method to compute limit invariant distributions. This method should be useful in many applications of Markov processes (such as methods of equilibrium selection in non-cooperative games (Kandori et al., 1993; Young, 1993) or models of network formation (Jackson and Watts, 2002).

Organization of the Paper The remainder of the paper is organized as follows. In Section 2, we define multiple-type housing markets. In Section 3, we discuss some basic results for multiple-type housing markets and introduce examples. In Section 4, we introduce the d.r. process and characterize its recurrent classes. In Section 5, we continue with the perturbed d.r. process and study stochastic stability and the limit invariant distribution. Finally, Section 6 concludes with some remarks on the relation between the different solution concepts.

\section{Multiple-Type Housing Markets: the Model}

Let $N=\{1, \ldots, n\}, n \geq 2$, be the set of agents, which we sometimes call the grand coalition. There exist $\bar{\ell} \geq 1$ types of indivisible objects. The set of object types is denoted by $L=$ $\{1, \ldots, \bar{\ell}\}$ and each agent $i \in N$ is endowed with one object of each type $\ell \in L$, denoted by $i$. Thus, $N$ also denotes the set of objects of each type. 
Allocations An allocation is an assignment of objects such that each agent receives exactly one object of each type, i.e., an allocation is a matrix $x=\left(x_{i}(\ell)\right)_{i \in N, \ell \in L} \in N^{N \times L}$ such that

(i) For each $i \in N$ and each $\ell \in L, x_{i}(\ell) \in N$ denotes the object of type $\ell$ that agent $i$ consumes, e.g., if $x_{i}(\ell)=j$, then agent $i$ receives agent $j$ 's endowment of type $\ell$, and

(ii) no object of any type is assigned to more than one agent, i.e., for each $\ell \in L$, $\cup_{i \in N}\left\{x_{i}(\ell)\right\}=N$.

Let $X$ denote the set of allocations. Given $x \in X$ and $\ell \in L, x(\ell)=\left(x_{1}(\ell), \ldots, x_{n}(\ell)\right)$ denotes the allocation of type- $\ell$ objects. Given $x \in X$ and $i \in N, x_{i}=\left(x_{i}(1), \ldots, x_{i}(\bar{\ell})\right)$ denotes the list of objects that agent $i$ receives at allocation $x$. We call $x_{i}$ agent $i$ 's bundle. Note that the set of bundles for each $i \in N$ can be denoted by $N^{L}$. We denote each agent $i$ 's endowment by $e_{i}=(i, \ldots, i) \in N^{L}$. Similarly, for any coalition $S \subseteq N$, we denote coalition $S$ 's endowment by $e_{S}=\left(e_{i}\right)_{i \in S}$.

Markets Each agent $i \in N$ has complete, transitive, and strict preferences $R_{i}$ over bundles, i.e., $R_{i}$ is a linear order over $N^{L}$. We denote the strict part of $R_{i}$ by $P_{i}$. Thus, for bundles $x_{i}, y_{i} \in N^{L}, x_{i} R_{i} y_{i}$ implies $\left[x_{i} \neq y_{i}\right.$ and $\left.x_{i} P_{i} y_{i}\right]$ or $\left[x_{i}=y_{i}\right]$. By $\mathcal{R}$ we denote the set of preferences over $N^{L}$. By $\mathcal{R}^{N}=\times_{i \in N} \mathcal{R}$ we denote the set of (preference) profiles. Since the set of agents and their endowments remain fixed throughout, $\mathcal{R}^{N}$ also denotes the set of multiple-type housing markets. For $\bar{\ell}=1$, our model coincides with the classical Shapley and Scarf (1974) housing market model. ${ }^{6}$

Individual Rationality An allocation $x$ is individually rational for $R \in \mathcal{R}^{N}$ if for each $i \in N, x_{i} R_{i} e_{i}$. Let $I R(R)$ be the set of individually rational allocations for $R \in \mathcal{R}^{N}$.

To introduce the standard (cooperative) solution concepts for multiple-type housing markets we need some additional notation. The set of all feasible reallocations of objects among the members of coalition $S \subseteq N$ is denoted by,

$$
X_{S}=\left\{\left(x_{i}(\ell)\right)_{i \in S, \ell \in L} \in N^{S \times L} \mid \text { for each } \ell \in L, \cup_{i \in S}\left\{x_{i}(\ell)\right\}=S\right\} .
$$

Let $y \in X$ and $S \subseteq N$. Then, by $y_{S}=\left(y_{i}\right)_{i \in S}$ we denote the restriction of allocation $y$ to coalition $S$. For notational convenience we will also use $X_{-S} \equiv X_{N \backslash S}$ and $y_{-S} \equiv y_{N \backslash S}$.

If for $R \in \mathcal{R}^{N}, x, y \in X$, and $S \subseteq N$, (i) $y_{S} \in X_{S}$, (ii) for each $i \in S, y_{i} R_{i} x_{i}$, and (iii) for some $j \in S, y_{j} P_{j} x_{j}$, then $y S$-blocks $x$.

The Core An allocation $x \in X$ is a (strong) core allocation for $R \in \mathcal{R}^{N}$ if there exists no coalition $S \subseteq N$ and no $y_{S} \in X_{S}$ such that $y S$-blocks $x$. Let $\operatorname{Core}(R)$ be the set of core allocations - the core - for $R \in \mathcal{R}^{N}$.

Walrasian Allocations Define a price system by $p \equiv\left(p_{\ell}\right)_{\ell \in L} \in \mathbb{R}_{+}^{n \bar{\ell}}$ such that for all $l \in L$, $p_{\ell}=\left(p_{\ell}(1), \ldots, p_{\ell}(n)\right) \in \mathbb{R}_{+}^{n}$. An allocation $x$ is a Walrasian allocation for $R \in \mathcal{R}^{N}$ if there exists a price system $p \in \mathbb{R}_{+}^{n \bar{\ell}} \backslash\{0\}$ such that for each $i \in N, x$ is a best affordable bundle, i.e., (i) $\sum_{\ell \in L} p_{\ell}(i) \geq \sum_{\ell \in L} p_{\ell}\left(x_{i}(\ell)\right)$ and (ii) if $y_{i} P_{i} x_{i}$, then $\sum_{\ell \in L} p_{\ell}\left(y_{i}(\ell)\right)>\sum_{\ell \in L} p_{\ell}(i) .{ }^{7}$ Let $W(R)$ be the set of Walrasian allocations - the Walrasian set - for $R \in \mathcal{R}^{N}$.

\footnotetext{
${ }^{6}$ Note that instead of considering the whole domain of linear orders $\mathcal{R}$ as our reference domain, we could restrict the domain to the domain of separable preferences $\mathcal{R}^{s}$ (see Klaus, 2006) or to the domain of additively separable preferences $\mathcal{R}^{\text {as }}$ (see Konishi et al., 2001). However, separability plays no role in our analysis.

${ }^{7}$ Note that the budget inequality (i) can be replaced by a budget equality: this can be easily checked by adding (i) up over all agents $i \in N$ and applying $\sum_{i \in N}\left(\sum_{\ell \in L} p_{\ell}(i)\right)=\sum_{i \in N}\left(\sum_{\ell \in L} p_{\ell}\left(x_{i}(\ell)\right)\right)$.
} 


\section{Multiple-Type Housing Markets: Basic Results \& Examples}

First, we summarize some results for the benchmark case of one object type housing markets.

\section{Remark 1. The Benchmark Case: Housing Markets with Strict Preferences}

For any housing market with one object type (and strict preferences) a core allocation always exists (Shapley and Scarf, 1974). Furthermore, the core equals the Walrasian set and is a singleton (Roth and Postlewaite, 1977). Using the so-called top-trading algorithm (due to David Gale, see Shapley and Scarf, 1974) one can easily calculate the unique core allocation for any housing market with one object type. Furthermore, the core is externally stable, i.e., for any non-core allocation $x$ there exists a coalition $S$ such that the core allocation $S$-blocks $x$ (Roth and Postlewaite, 1977). Serrano and Volij (2005) refer to this particular feature of the core as "global dominance."

As soon as we either relax the assumption of strict preferences or increase the number of object types, existence, single-valuedness, and the global dominance property of the core fail. For markets with $\bar{\ell} \geq 2$, the core may be empty or multi-valued - even for additively separable preferences (Konishi et al., 2001). Moreover, for each $R \in \mathcal{R}^{N}, W(R) \subseteq \operatorname{Core}(R)$ (Konishi et al., 2001, Proposition 3.1).

We next introduce several examples that we will analyze in the sequel. ${ }^{8}$ All our examples are multiple-type housing market with two object types and three agents.

Example 1. An Empty Core Let $R$ be such that

$$
\begin{aligned}
& R_{1}:(3,1),(1,2),(1,1), \text { anything, } \\
& R_{2}:(2,1),(3,2),(2,2), \text { anything, } \\
& R_{3}:(2,3),(1,3),(3,3), \text { anything. }
\end{aligned}
$$

The set $\operatorname{IR}(R)$ of individually rational allocations is $\left\{x^{1}, x^{2}, x^{3}, x^{4}\right\}$, with

$$
\begin{aligned}
& x^{1}=\{(1,1),(2,2),(3,3)\}, \quad x^{2}=\{(1,1),(3,2),(2,3)\}, \quad x^{3}=\{(1,2),(2,1),(3,3)\}, \\
& x^{4}=\{(3,1),(2,2),(1,3)\} .
\end{aligned}
$$

Clearly, $x^{2}\{2,3\}$-blocks $x^{1}, x^{3}\{1,2\}$-blocks $x^{2}, x^{4}\{1,3\}$-blocks $x^{3}$, and $x^{2}\{2,3\}$-blocks $x^{4}$. Hence, $\operatorname{Core}(R)=W(R)=\emptyset$.

We relegate the computation of the core and the Walrasian set for all remaining examples to Appendix A.

\section{Example 2. The Unique Walrasian Allocation Equals the Core Allocation}

Let $R$ be such that

$$
\begin{aligned}
& R_{1}:(3,1),(2,2),(1,2),(1,1), \text { anything, } \\
& R_{2}:(2,1),(3,3),(3,2),(2,2), \text { anything, } \\
& R_{3}:(2,3),(1,1),(1,3),(3,3), \text { anything. }
\end{aligned}
$$

\footnotetext{
${ }^{8}$ The exception is Example 5, which we comment on in Remark 2.
} 
The set $\operatorname{IR}(R)$ of individually rational allocations is $\left\{x^{1}, x^{2}, x^{3}, x^{4}, x^{5}\right\}$, with

$$
\begin{aligned}
& x^{1}=\{(1,1),(2,2),(3,3)\}, \quad x^{2}=\{(1,1),(3,2),(2,3)\}, \quad x^{3}=\{(1,2),(2,1),(3,3)\}, \\
& x^{4}=\{(3,1),(2,2),(1,3)\}, \quad x^{5}=\{(2,2),(3,3),(1,1)\} .
\end{aligned}
$$

Furthermore, Core $(R)=W(R)=\left\{x^{5}\right\}$.

The next example has multiple core allocations, of which only one is Walrasian. ${ }^{9}$

\section{Example 3. Multiple Core Allocations and a Unique Walrasian Allocation}

Let $R$ be such that

$$
\begin{aligned}
& R_{1}:(1,2),(3,3),(2,3),(1,1), \text { anything, } \\
& R_{2}:(1,3),(1,2),(3,3),(2,2), \text { anything, } \\
& R_{3}:(3,1),(2,1),(3,3), \text { anything. }
\end{aligned}
$$

The set $\operatorname{IR}(R)$ of individually rational allocations is $\left\{x^{1}, x^{2}, x^{3}, x^{4}\right\}$, with

$$
\begin{aligned}
& x^{1}=\{(1,1),(2,2),(3,3)\}, \quad x^{2}=\{(2,3),(1,2),(3,1)\}, \quad x^{3}=\{(1,2),(3,3),(2,1)\}, \\
& x^{4}=\{(3,3),(1,2),(2,1)\} .
\end{aligned}
$$

Furthermore, $W(R)=\left\{x^{3}\right\} \varsubsetneqq\left\{x^{2}, x^{3}, x^{4}\right\}=\operatorname{Core}(R)$.

Next we illustrate that the Walrasian set may contain multiple allocations.

Example 4. Multiple Walrasian Allocations Let $R$ be such that

$R_{1}:(1,2),(3,3),(2,3),(1,1)$, anything,

$R_{2}:(1,3),(1,2),(3,3),(2,2)$, anything

$R_{3}:(2,1),(3,1),(3,3)$, anything.

The set $\operatorname{IR}(R)$ of individually rational allocations is $\left\{x^{1}, x^{2}, x^{3}, x^{4}\right\}$, with

$$
\begin{aligned}
& x^{1}=\{(1,1),(2,2),(3,3)\}, \quad x^{2}=\{(2,3),(1,2),(3,1)\}, \quad x^{3}=\{(3,3),(1,2),(2,1)\}, \\
& x^{4}=\{(1,2),(3,3),(2,1)\} .
\end{aligned}
$$

Furthermore, $W(R)=\operatorname{Core}(R)=\left\{x^{3}, x^{4}\right\}$.

In our last example the Walrasian set is empty while the core is nonempty.

Example 5. No Walrasian Allocation and a Multi-Valued Core Let $R$ be such that $R_{1}:(1,2),(3,3),(2,3),(1,1)$, anything, $R_{2}:(3,2),(1,2),(3,3),(2,2)$, anything,

\footnotetext{
${ }^{9}$ Since in our context, separability does not play a role, we introduce alternative examples to the ones analyzed in Konishi et al. (2001); for instance, Example 3 has four individually rational allocations while Konishi et al.'s (2001) corresponding example has eleven individually rational allocations.
} 


$$
R_{3}:(1,3),(3,1),(2,3),(2,1),(3,3), \text { anything. }
$$

The set $I R(R)$ of individually rational allocations is $\left\{x^{1}, x^{2}, x^{3}, x^{4}, x^{5}\right\}$, with

$$
\begin{aligned}
& x^{1}=\{(1,1),(2,2),(3,3)\}, \quad x^{2}=\{(1,1),(3,2),(2,3)\}, \quad x^{3}=\{(2,3),(1,2),(3,1)\}, \\
& x^{4}=\{(3,3),(1,2),(2,1)\}, \quad x^{5}=\{(1,2),(3,3),(2,1)\} .
\end{aligned}
$$

Furthermore, $W(R)=\emptyset \varsubsetneqq\left\{x^{2}, x^{3}\right\}=\operatorname{Core}(R)$.

The last two examples illustrate two features of the relationship between the core and the Walrasian set not yet recognized in the literature.

Remark 2. New Insights on Walrasian Allocations through Examples 4 and 5 Konishi et al. (2001) prove that the Walrasian set is a subset of the core and that it might be empty if the core is also empty. Example 5 shows that the Walrasian set might even be empty when the core is nonempty. Second, in Example 4 the Walrasian set contains more than one allocation.

Moreover, the examples collected in this section demonstrate that the core, or the Walrasian set, are not necessarily satisfactory solutions for multiple-type housing markets.

Remark 3. Core/Walrasian Allocations as Solutions? First, note that both standard static solution sets, the core and the Walrasian set, may be empty. When they are, we do not have any (static) prediction to offer as to what will happen. Will agents keep their endowments or will they trade? Second, the core and the Walrasian set may be multi-valued. When they are, again we cannot make (static) predictions which, if any, of the possible allocations in the core will result from trade. As discussed in the Introduction, we will explicitly model the dynamic aspect of the core through a dynamic recontracting process. Doing so will enable us to say more about core and Walrasian allocations as solutions of dynamic trade.

Next, we model the dynamic recontracting process and characterize market outcomes via the respective set of recurrent classes (Section 4), stochastic stability (Section 5.1), and - last but not least - the limit invariant distribution (Section 5.2).

\section{Unperturbed Dynamic Recontracting Processes}

For each $R \in \mathcal{R}^{N}$, dynamic recontracting is modeled by a Markov Process $(X, M(R)){ }^{3}$ The state space is the set of allocations $X$ and $M(R)$ is a transition matrix that describes the following dynamics. In each period $t$, the process is at an allocation $x(t) \in X$ and a coalition $S$ of agents is randomly selected. The process moves from $x(t)$ to another allocation $x(t+1)$ when the agents in $S$ recontract among themselves, i.e., agree upon a redistribution of their endowments. Agents recontract if they benefit from doing so by means of blocking - they don't make mistakes. As we will allow for mistakes or perturbations later on, we refer to the dynamic recontracting process discussed in this section as unperturbed. The following three assumptions are satisfied in each period.

\section{Assumption 1. Opportunities to Recontract}

Each coalition $S \subseteq N$ is chosen with positive probability to recontract. A coalition that has this opportunity is an active coalition. 
This rather mild assumption covers most of the common models of coalition formation. In particular, the probability with which a certain coalition has the opportunity to recontract can depend on the size of the coalition, the allocation in period $t$, or the identities of the agents involved as long as the corresponding probability distribution has full support in the set of coalitions.

For each $S \subseteq N$ and each $x \in X$, let $B_{S}(x)=\left\{y_{S} \in X_{S} \mid\right.$ for each $i \in S, y_{i} R_{i}$ $x_{i}$ and for some $\left.j \in S, y_{j} P_{j} x_{j}\right\}$ be the set of $S$-blockings of $x$.

\section{Assumption 2. Recontracting Behavior}

Given $x(t)$, an active coalition $S \subseteq N$ recontracts if $B_{S}(x(t)) \neq \emptyset$. If $\left|B_{S}(x(t))\right|>1$, then each $y_{S} \in B_{S}(x(t))$ is chosen with positive probability.

Agents are myopic in the sense that they agree upon a reallocation of their endowments if it is weakly improving in the subsequent period, even though they might be worse off later.

The assumption according to which weakly improving reallocations are chosen is again mild. It is only important that any such reallocation is chosen with positive probability. The probability itself can depend on the identities or even the preferences of the agents in the active coalition.

We now fix the allocation that results from recontracting.

\section{Assumption 3. Allocations Resulting from Recontracting}

Let $S$ be the active coalition in period $t$. If $B_{S}(x(t))=\emptyset$, then $x(t+1)=x(t)$. Let $y_{S} \in B_{S}(x(t))$ be the blocking chosen by $S$. If $x_{-S}(t) \in X_{-S}$, then $x(t+1)=\left(y_{S}, x_{-S}(t)\right)$, and otherwise, $x(t+1)=\left(y_{S}, e_{S}\right)$.

A Markov process $(X, M(R))$ that satisfies Assumptions 1, 2, and 3 is an unperturbed dynamic recontracting process, or u.d.r. process for short. Serrano and Volij (2005, Section 7$)$ consider this specification of the u.d.r. process for Shapley-Scarf economies $(\bar{\ell}=1)$.

An important solution for the u.d.r. process is the set of recurrent classes. A set $A \subseteq X$ is a recurrent class if it is a minimal set of allocations that once entered throughout the u.d.r. process is never left.

Let $R \in \mathcal{R}^{N}$. We denote the set of recurrent sets by $\mathcal{R S}(R)=\{A \subseteq X \mid$ for each $x \in$ $A$ and each $\left.x^{\prime} \notin A, M(R)\left(x, x^{\prime}\right)=0\right\}$. The set of recurrent classes $\mathcal{R C}(R)$ is the set of all minimal recurrent sets. Notice that $\mathcal{R C}(R)$ is a set of sets of allocations. It is convenient to also denote the set of recurrent allocations by $R C(R)=\{x \in X \mid$ there exists $A \in$ $\mathcal{R C}(R)$ with $x \in A\}$.

Theorem 1. Recurrent Classes

Let $R \in \mathcal{R}^{N}$ and $(X, M(R))$ be an u.d.r. process. Then,

(i) $R C(R) \neq \emptyset$.

(ii) $\{x\} \in \mathcal{R C}(R) \Leftrightarrow x \in \operatorname{Core}(R)$.

(iii) $W(R) \subseteq \operatorname{Core}(R) \subseteq R C(R) \subseteq I R(R) \cdot{ }^{10}$

\footnotetext{
${ }^{10}$ Example 2 is a multiple-type housing market with $\operatorname{Core}(R) \subsetneq R C(R) \subsetneq I R(R)$ and Example 3 is a multiple-type housing market with $W(R) \subsetneq \operatorname{Core}(R)$.
} 
Proof. Statement (i) follows from the finiteness of the Markov process (the finiteness of $X$ ).

Statement (ii) is a direct implication of the definition of the u.d.r. process and the fact that a core allocation cannot be blocked by any other allocation.

Statement (iii): $W(R) \subseteq$ Core $(R)$ follows from Konishi et al. (2001, Proposition 3.1). Statement (ii) implies $\operatorname{Core}(R) \subseteq R C(R)$. Note that, by the definition of the u.d.r. process, in each period $t, x(t) \in I R(R)$. Hence, $R C(R) \subseteq I R(R)$.

Theorem 1 shows that the set of recurrent allocations of an u.d.r. process deserves attention as a solution for multiple-type housing markets: for any multiple-type housing market the set of recurrent allocations is non-empty, consists only of individually rational allocations, and contains all core allocations. To demonstrate (i) the non-emptiness of $R C(R)$, (ii) the coexistence of singleton recurrent classes (i.e., allocations in the core) and non-singleton recurrent classes, and (iii) the absence of selection between core allocations, we continue with three of the examples introduced in Section $3 .^{11}$

In Example 1 we observe a multi-valued (non-core) recurrent class.

\section{Example 1 (continued). Empty Core and Non-Singleton Recurrent Class}

Recall that $\operatorname{Core}(R)=\emptyset$. By Theorem 1(iii), only allocations in $\operatorname{IR}(R)=\left\{x^{1}, x^{2}, x^{3}, x^{4}\right\}$ can be recurrent allocations. Since $x^{1}$ can be blocked by any other allocation in $\operatorname{IR}(R)$, it can never be an element of a recurrent class. Hence, we are left with $x^{2}, x^{3}$, and $x^{4}$. Recall that we have a blocking cycle where $x^{3}\{1,2\}$-blocks $x^{2}, x^{4}\{1,3\}$-blocks $x^{3}$, and $x^{2}\{2,3\}$-blocks $x^{4}$. Thus, none of $x^{2}, x^{3}$, and $x^{4}$ can form a singleton recurrent class. Furthermore, each of these allocations can be reached from one another through (a sequence of) blocking(s) while once one of these allocations is reached no outside allocation can block. Therefore, $\left\{x^{2}, x^{3}, x^{4}\right\}$ constitutes the only recurrent class. Hence, $\mathcal{R C}(R)=\left\{\left\{x^{2}, x^{3}, x^{4}\right\}\right\}$ and $R C(R)=\left\{x^{2}, x^{3}, x^{4}\right\}$.

In Example 2 we have two recurrent classes (one of them equals the core and contains the unique Walrasian allocation, the other contains three non-core allocations).

Example 2 (continued). The Set of Recurrent Classes Exceeds the Core Recall that $\operatorname{Core}(R)=\left\{x^{5}\right\}$. By Theorem 1(iii), only allocations in $I R(R)=$ $\left\{x^{1}, x^{2}, x^{3}, x^{4}, x^{5}\right\}$ can be recurrent allocations. By Theorem 1(ii), Core $(R)=\left\{x^{5}\right\}$ is the only singleton recurrent class. Next, recall that the only difference between Examples 1 and 2 is that - loosely speaking - we added allocation $x^{5}$ to the agents' preferences such that $x^{5}$ is now not only individually rational, but also the unique core allocation. However, none of $x^{2}, x^{3}$, or $x^{4}$ can be blocked by $x^{5}$ (or $x^{1}$ ) while there is again the blocking cycle where $x^{3}$ $\{1,2\}$-blocks $x^{2}, x^{4}\{1,3\}$-blocks $x^{3}$, and $x^{2}\{2,3\}$-blocks $x^{4}$. Thus, $\left\{x^{2}, x^{3}, x^{4}\right\}$ forms (as in Example 1) the only non-singleton recurrent class. Hence, $\mathcal{R C}(R)=\left\{\left\{x^{2}, x^{3}, x^{4}\right\},\left\{x^{5}\right\}\right\}$ and $R C(R)=\left\{x^{2}, x^{3}, x^{4}, x^{5}\right\}$.

We conclude with a multiple-type housing market where the set of recurrent classes coincides with the set of core allocations.

\section{Example 3 (continued). The Core Equals the Set of Recurrent Classes}

Recall that $\operatorname{Core}(R)=\left\{x^{2}, x^{3}, x^{4}\right\}$. By Theorem 1(iii), only allocations in $\operatorname{IR}(R)=$ $\left\{x^{1}, x^{2}, x^{3}, x^{4}\right\}$ can be recurrent allocations. Since $x^{1}$ can be blocked by any other allocation in $\operatorname{IR}(R)$, it can never be an element of a recurrent class. With Theorem 1(ii),

\footnotetext{
${ }^{11}$ The recurrent classes of the other examples are determined in Appendix A.
} 
$\left\{x^{2}\right\},\left\{x^{3}\right\}$ and $\left\{x^{4}\right\}$ are the only recurrent classes. Hence, $\mathcal{R C}(R)=\left\{\left\{x^{2}\right\},\left\{x^{3}\right\},\left\{x^{4}\right\}\right\}$ and $R C(R)=\operatorname{Core}(R)$.

To summarize, even in the case of an empty core, $R C(R)$ offers a prediction for the outcome of a multiple-type housing market (Example 1). This, however, is achieved at the expense of a weakly larger set of final outcomes whenever the core is non-empty (Example 2). Note that this closely resembles the situation in the literature on evolutionary selection of Nash equilibria in, e.g., coordination games (Young, 1993): while every Nash equilibrium of the coordination game is also a singleton recurrent class of the unperturbed learning process, the set of recurrent classes typically exceeds the set of Nash equilibria.

\section{$5 \quad$ Perturbed Dynamic Recontracting Processes}

We will now perturb the dynamic recontracting process by allowing agents to make mistakes, i.e., an agent might agree on a reallocation that makes him worse off. We follow the standard approach by assuming that in any given period, any agent in an active coalition can make a mistake with probability $\epsilon>0$. We restrict perturbations to mistakes where agents agree on individually rational allocations that make them worse off. ${ }^{12}$ One could argue that any active coalition knows that an individually irrational block is never sustainable because an agent who receives a bundle that is worse than his endowment later (with positive probability) is allowed to recontract with himself and then has the good sense to improve on his bundle by enforcing his endowment.

From now on we assume that the state space for any $R \in \mathcal{R}$ equals the set of individually rational allocations, i.e., $X=I R(R)$.

Assumption 4. The probability with which a member of an active coalition $i \in S$ agrees on a reallocation $y_{S}$ with $x_{i}(t) P_{i} y_{i} R_{i} e_{i}$ equals $\epsilon>0$.

In particular, the probability of a mistake does not depend on the active coalition, the given $x(t)$, or agents' preferences. A Markov process $\left(X, M^{\epsilon}(R)\right)$ that satisfies Assumptions 1 -4 is a perturbed dynamic recontracting process, or p.d.r. process for short. The p.d.r. process is ergodic: as mistakes induce (indirect) transitions between any two allocations its unique recurrent class is the entire state space $X=I R(R)$ (i.e., the p.d.r. process is irreducible). Then, the p.d.r. process exhibits a unique invariant distribution $\mu_{\epsilon}(R)$ - for notational convenience from now on simply $\mu_{\epsilon}$ - with support $I R(R)$ that displays the long-run probability distribution over allocations (i.e., for any $x \in X, \mu_{\epsilon}(x)$ is the probability that the process will be at allocation $x$ in the long-run). Moreover, the perturbation is regular on $\operatorname{IR}(R)$, i.e., transition probabilities between any two individually rational allocations are non-zero and polynomials in $\epsilon$. As every transition from an allocation $x^{\prime}$ to an allocation $x^{\prime \prime}$ can involve a maximum of $n$ mistakes (by every agent in $N$ ) we can denote $M^{\epsilon}(R)\left(x^{\prime}, x^{\prime \prime}\right) \equiv \sum_{k=0, \ldots, n} m_{k}\left(x^{\prime}, x^{\prime \prime}\right) \epsilon^{k}$ where $m_{k}\left(x^{\prime}, x^{\prime \prime}\right)$ captures the probability that a coalition forms at allocation $x^{\prime}$ and agrees upon allocation $x^{\prime \prime}$ with exactly $k$ agents making a mistake. In particular, $m_{0}\left(x^{\prime}, x^{\prime \prime}\right)=M(R)\left(x^{\prime}, x^{\prime \prime}\right)$ (the intercept of the polynomial) is the respective entry in the transition matrix of the u.d.r. process.

\footnotetext{
${ }^{12}$ The consequences of also allowing "individually irrational mistakes" would be a more cumbersome analysis of the examples in Subsection 5.2. Preferences would have to be specified over all bundles (also the individually irrational ones) and taken into account in the construction of least resistance trees (see below). However, it is easy to see that the general results described in Theorems 2 and 3 still hold.
} 
The limit invariant distribution $\mu^{*}$ of a Markov process $(X, M(R))$ is the (unique) invariant distribution $\mu_{\epsilon}$ of a perturbed process $\left(X, M^{\epsilon}(R)\right.$ ) in the limit of $\epsilon \rightarrow 0$ (i.e., $\mu^{*} \equiv \lim _{\epsilon \rightarrow 0} \mu_{\epsilon}$ ). Hence, $\mu^{*}(x)$ depicts the probability that the process will be at allocation $x$ in the long-run and in the limit of vanishing mistakes. Young (1993, Theorem 4(i)) shows that the limit invariant distribution exists and that it is an invariant distribution of the u.d.r. process $(X, M(R))$ if the perturbation is regular (i.e., if Assumption 4 holds). The support of every invariant distribution of the unperturbed process is a (non-empty) collection of its recurrent classes. Allocations in the support of $\mu^{*}$ are called stochastically stable. Hence, if an allocation $x$ is stochastically stable, the p.d.r. process will be at $x$ in the long run and in the limit of vanishing mistakes with strictly positive probability (i.e., $\mu^{*}(x)>0$ ). We denote the set of stochastically stable allocations by $S R C(R)$.

We first explore stochastic stability for our d.r. processes.

\subsection{Stochastic Stability}

Lemma 1. Young (1993, Theorem 4(i)) Let $R \in \mathcal{R}^{N}$ and $(X, M(R))$ be an u.d.r. process. Then,

$$
S R C(R) \neq \emptyset \text { and } S R C(R) \subseteq R C(R) .
$$

Next, for each $R \in \mathcal{R}$, we introduce a general methodology to determine the set of stochastically stable allocations $S R C(R)$ and apply it to our examples.

$x$-Trees Consider the set of directed graphs that have vertex set $X=I R(R)$. Then, any directed graph is defined by its set of directed edges. We denote a directed edge from $x^{\prime}$ to $x^{\prime \prime}$ by $\left[x^{\prime}, x^{\prime \prime}\right]$ and interpret it as $x^{\prime \prime}$ is the outcome of recontracting that started from $x^{\prime}$. Note that the irreducibility of the p.d.r. process implies that for any directed edge $\left[x^{\prime}, x^{\prime \prime}\right]$ we have that $M^{\epsilon}(R)\left(x^{\prime}, x^{\prime \prime}\right)>0$. An $x$-tree $T_{x}$ (or a spanning tree) is a directed graph such that for every $y \in X$ with $y \neq x$ there is exactly one (cycle-free) sequence of edges (a directed path) from $y$ to $x$. Denote by $\mathcal{T}_{x}$ the set of all $x$-trees.

Stochastic Potential Let $\left[x^{\prime}, x^{\prime \prime}\right]$ be an edge in an $x$-tree $T_{x} \in \mathcal{T}_{x}$. The edge-resistance $r\left(x^{\prime}, x^{\prime \prime}\right)$ is the minimum number of mistakes needed to get directly from $x^{\prime}$ to $x^{\prime \prime}$, i.e., the minimal number of agents that are worse off through recontracting when actively participating in a blocking of allocation $x^{\prime}$ that results in allocation $x^{\prime \prime}$. Formally, $r\left(x^{\prime}, x^{\prime \prime}\right)=\min \{r \geq 0 \mid \infty>$ $\left.\lim _{\epsilon \rightarrow 0} \epsilon^{-r} M^{\epsilon}(R)\left(x^{\prime}, x^{\prime \prime}\right)>0\right\}$. Finally, the stochastic potential of $x \in X$, denoted by $\gamma(x)$, is the minimal sum of edge-resistances over all $x$-trees, i.e., $\gamma(x)=\min _{T_{x} \in \mathcal{T}_{x}} \sum_{\left[x^{\prime}, x^{\prime \prime}\right] \in T_{x}} r\left(x^{\prime}, x^{\prime \prime}\right)$. An $x$-tree $\widetilde{T}_{x}$ that minimizes $\sum_{\left[x^{\prime}, x^{\prime \prime}\right] \in T_{x}} r\left(x^{\prime}, x^{\prime \prime}\right)$ is a least resistance $x$-tree.

Stochastic Stability An allocation $x$ is stochastically stable if and only if it minimizes the stochastic potential $\gamma(x)$. The set of stochastically stable allocations $S R C(R)$ can be characterized as follows.

Lemma 2. Young (1993, Theorem 4(ii)) Let $R \in \mathcal{R}^{N}$ and $(X, M(R))$ be an u.d.r. process. Then, allocation $x \in X$ is stochastically stable if and only if for all $y \in X, \gamma(x) \leq \gamma(y)$.

Interpretation and Basic Implications The set of recurrent classes of a dynamic recontracting process are internally stable because no allocation in a recurrent class can be blocked by an "outside allocation". In the case of singleton recurrent classes this internal stability 
requirement coincides with the (internal) stability of the core. Stochastic stability also re-

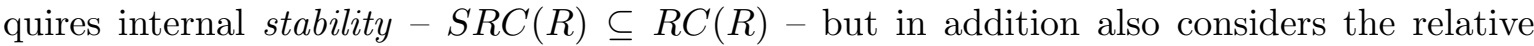
accessibility of recurrent classes, i.e., the number of mistakes agents need to make in order to reach a recurrent class from all other allocations. This notion of accessibility is captured by the stochastic potential. By also imposing a measure of accessibility, stochastic stability might serve as a selection device among recurrent classes and among core allocations.

Note that allocations in the same recurrent class have the same stochastic potential. To see this consider two allocations $x$ and $y$ in the same recurrent class. Now take any least resistance $x$-tree $\widetilde{T}_{x}$. Obviously, there exists a path from $y$ to $x$ consisting solely of edges with zero resistance, and such a path has to be part of $\widetilde{T}_{x}$ (otherwise $\widetilde{T}_{x}$ would not be a least resistance tree). Likewise, there also exists a path from $x$ to $y$ consisting solely of edges with zero resistance. But then we can construct a $y$-tree $\widetilde{T}_{y}$ with $\sum_{\left[x^{\prime}, x^{\prime \prime}\right] \in \widetilde{T}_{x}} r\left(x^{\prime}, x^{\prime \prime}\right)=$ $\sum_{\left[x^{\prime}, x^{\prime \prime}\right] \in \widetilde{T}_{y}} r\left(x^{\prime}, x^{\prime \prime}\right)$ which implies that $\gamma(x) \geq \gamma(y)$. Graphically one can obtain the $y$-tree $\widetilde{T}_{y}$ by first drawing the zero resistance path from $x$ to $y$ and then attaching the missing vertices by using only branches of $\widetilde{T}_{x}$ - thus $\widetilde{T}_{y}$ is obtained from $\widetilde{T}_{x}$ by "tree surgery". As the argument to obtain $\gamma(x) \geq \gamma(y)$ is symmetric with respect to $x$ and $y$, it follows that $\gamma(x)=\gamma(y)$. Because of the identity of stochastic potentials within a given recurrent class, we simply refer to $\gamma(x)$ as the stochastic potential of the recurrent class that includes $x$.

Observe, however, that the stochastic potential is only determined by the resistance of a tree in the set of least resistance trees. Consider, for instance, $R \in \mathcal{R}$ with $\mathcal{R C}(R)=$ $\{\{x\},\{y\}\}$. To establish stochastic stability of $\{x\}$, we only have to find one $x$-tree with treeresistance $\gamma(y)$. The number of least resistance trees for either $\{x\}$ or $\{y\}$ does not influence the result. This already suggests that the stochastic potential does not capture all aspects of accessibility and stability of allocations. We return to this observation in Subsection 5.2.

Computation Let $R \in \mathcal{R}^{N}$. Lemma 2 offers the following procedure to compute $S R C(R)$.

1. Determine the set of recurrent classes $\mathcal{R C}(R)$.

2. Let $A \in \mathcal{R C}(R)$ and $x \in A$. Construct a least resistance $x$-tree and compute $\gamma(x)$. For every $y \in A$, set $\gamma(y)=\gamma(x)$.

3. Determine $S R C(R)=\{x \in R C(R) \mid$ for all $y \in R C(R), \gamma(x) \leq \gamma(y)\}$.

To illustrate our methodology, we apply it to the examples discussed in Section 4.

Example 1 (continued). Empty Core and Non-Singleton $S R C(R)$

Recall that $\mathcal{R C}(R)=\left\{\left\{x^{2}, x^{3}, x^{4}\right\}\right\}$. Hence by Lemmas 1 and 2 , and the observation that allocations in the same recurrent class have the same stochastic potential, $S R C(R)=$ $\left\{x^{2}, x^{3}, x^{4}\right\}=R C(R)$.

The following examples illustrate how to compute the stochastic potential.

Example 2 (continued). $S R C(R)=R C(R) \nsupseteq \operatorname{Core}(R)$

Recall that $\mathcal{R C}(R)=\left\{\left\{x^{2}, x^{3}, x^{4}\right\},\left\{x^{5}\right\}\right\}$. All allocations in $\left\{x^{2}, x^{3}, x^{4}\right\}$ have the same stochastic potential. Hence, it is sufficient to construct a least resistance $x^{2}$-tree. Note that $x^{2}$ can be reached from $x^{1}, x^{3}$, and $x^{4}$ without mistakes. Thus, we only have to link up $x^{5}$ with a minimal number of mistakes. Every allocation in $\left\{x^{2}, x^{3}, x^{4}\right\}$ can be reached from $x^{5}$ with one mistake, e.g., $\{2,3\}$ can recontract to obtain $x^{2}$ with a mistake by agent 2 . Therefore $\widetilde{T}_{x^{2}}=\left\{\left[x^{1}, x^{2}\right],\left[x^{4}, x^{2}\right],\left[x^{3}, x^{4}\right],\left[x^{5}, x^{2}\right]\right\}$ is a least resistance $x^{2}$-tree and $\gamma\left(x^{2}\right)=\gamma\left(x^{3}\right)=\gamma\left(x^{4}\right)=1$. 
The grand coalition can recontract at any of the allocations $x^{2}, x^{3}$, or $x^{4}$ to reach $x^{5}$ with one mistake, e.g., starting from $x^{2}$ the grand coalition can recontract to obtain $x^{5}$ with a mistake by agent 3 . Note that $x^{2}$ can be reached from $x^{1}, x^{3}$, and $x^{4}$ without mistakes. Therefore $\widetilde{T}_{x^{5}}=\left\{\left[x^{4}, x^{2}\right],\left[x^{3}, x^{4}\right],\left[x^{2}, x^{5}\right],\left[x^{1}, x^{2}\right]\right\}$ is a least resistance $x^{5}$-tree and $\gamma\left(x^{5}\right)=1$. Thus, $S R C(R)=\left\{x^{5}, x^{2}, x^{3}, x^{4}\right\}=R C(R)$.

Example 3 (continued). $\operatorname{SRC}(\boldsymbol{R})=\boldsymbol{R} C(R)=\operatorname{Core}(R)$ and $|\operatorname{Core}(R)|>1$

Recall that $\mathcal{R C}(R)=\left\{\left\{x^{2}\right\},\left\{x^{3}\right\},\left\{x^{4}\right\}\right\}$. From any of the core allocations, each single agent can recontract with himself to obtain $x^{1}$ by making one mistake. Note that any core allocation can be reached from $x^{1}$ without mistakes. Therefore, $\widetilde{T}_{x^{2}}=\left\{\left[x^{1}, x^{2}\right],\left[x^{3}, x^{1}\right],\left[x^{4}, x^{1}\right]\right\}$, $\widetilde{T}_{x^{3}}=\left\{\left[x^{1}, x^{3}\right],\left[x^{2}, x^{1}\right],\left[x^{4}, x^{1}\right]\right\}$, and $\widetilde{T}_{x^{4}}=\left\{\left[x^{1}, x^{4}\right],\left[x^{2}, x^{1}\right],\left[x^{3}, x^{1}\right]\right\}$ are least resistance trees, $\gamma\left(x^{2}\right)=\gamma\left(x^{3}\right)=\gamma\left(x^{4}\right)=2$, and $S R C(R)=\left\{x^{2}, x^{3}, x^{4}\right\}=R C(R)$.

The last example nicely illustrates a weakness of stochastic stability as a selection device for recurrent classes. Observe that a direct transition from $x^{2}$ to $x^{3}$ needs two mistakes while all other direct transitions between core allocations only ask for one mistake. This indicates that $x^{2}$ is more difficult to exit for the p.d.r. process than other core allocations, and $x^{3}$ is more difficult to access. This suggests $x^{2}$ as a more accessible and more stable allocation, or simply a better prediction for the final allocation. But due to the indirect paths from one recurrent class via $x^{1}$ to another recurrent class, all core allocations have a stochastic potential of 2 and the conjectured "superiority" of $x^{2}$ can not be established.

\section{Theorem 2. Stochastic Stability and Recurrent Classes}

Let $R \in \mathcal{R}^{N}$ and $(X, M(R))$ be an u.d.r. process. Then,

(i) for all $x \in R C(R), \gamma(x)=|\mathcal{R C}(R)|-1$ and

(ii) $R C(R)=S R C(R)$.

We first prove the following auxiliary result.

Claim 1. For all $x, x^{\prime} \in I R(R), x \neq x^{\prime}$, there is a path from $x$ to $x^{\prime}$ with at most one mistake.

Proof of Claim 1. We distinguish three cases. First, suppose that $x=e$. Since $x^{\prime}$ is individually rational, $r\left(x, x^{\prime}\right)=0$. Second, suppose that $x^{\prime}=e$. Then, $x \neq e$ and for at least one agent $i \in N, x_{i} \neq e_{i}$. When the singleton coalition $\{i\}$ forms, agent $i$ can claim back his endowment, which requires at most one mistake (by agent $i$ ). Since $x_{N \backslash\{i\}}$ is no longer feasible, all agents in $N \backslash\{i\}$ receive their endowments. Hence, $r\left(x, x^{\prime}\right) \leq 1$. Third, suppose $x \neq e$ and $x^{\prime} \neq e$. Then the previous findings imply that the sequence of edges $[x, e],\left[e, x^{\prime}\right]$ constitutes a path with $r(x, e)+r\left(e, x^{\prime}\right) \leq 1$, which concludes the proof of the claim.

Proof of Theorem 2. Using Claim 1, it is now easy to construct least-resistance trees.

Let $R \in \mathcal{R}$ and denote the respective (finite) set of recurrent classes by $\mathcal{R C}(R)=$ $\left\{A_{1}, \ldots, A_{m}\right\}$. It follows from the definition of a recurrent class that for all $x \in X, \gamma(x) \geq m-1$. Next, we prove that for all $x \in R C(R), \gamma(x) \leq m-1$.

For any allocation $x \in R C(R)$, we construct an $x$-tree as follows. Consider first the recurrent class $A_{k}$ with $x \in A_{k}$. By the definition of a recurrent class, for all $x^{\prime} \in A_{k}$, $r\left(x^{\prime}, x\right)=0$, i.e., there exists a set of edges that constitute the restriction of an $x$-tree to $A_{k}$ without mistakes. Suppose that all $x^{\prime} \in A_{k}$ are connected through that set of edges. Then pick another recurrent class $A_{l} \neq A_{k}$ and consider an allocation $y \in A_{l}$. Again, by the definition of a recurrent class, for all $y^{\prime} \in A_{l}, r\left(y^{\prime}, y\right)=0$ and there exists the restriction of a $y$-tree to $A_{l}$ without mistakes. Suppose that all $y^{\prime} \in A_{l}$ are connected through that set of 
edges. By Claim $1, r(y, x) \leq 1$. Hence, we can connect $A_{k}$ and $A_{l}$ with at most one mistake. So far we have constructed a "partial $x$-tree" that connects all allocations in $A_{k} \cup A_{l}$ with at most one mistake. Repeat this procedure for all remaining recurrent classes. In each step a new recurrent class is connected to the existing partial $x$-tree with at most one mistake. Hence, we can construct a complete $x$-tree with at most $m-1$ mistakes. Thus, $\gamma(x)=m-1$, which proves Statement (i). Statement (i) and Lemma 2 imply $S R C(R)=R C(R)$.

This result offers two insights. First, the requirement of stochastic stability does not work as a selection device for recurrent classes. In addition to coalitional stability as captured by the concept of a recurrent class, stochastic stability also incorporates a notion of coalitional accessibility (i.e., it compares the number of mistakes needed to reach an allocation in a certain recurrent class from all other recurrent classes) as captured in the stochastic potential. However, this notion of accessibility is rather limited as it only values the existence of least resistance trees, but e.g., not their number (see the different stability and accessibility features of allocations $x^{2}$ and $x^{3}$ in Example 3 ). In fact, Theorem 2 shows that this accessibility notion is too weak to distinguish between different recurrent classes.

Second, Theorem 2(ii) indicates that the selective power of stochastic stability as established by Serrano and Volij (2005) for housing markets with indifferences does not carry over to multiple-type housing markets with strict preferences - unlike one could have conjectured.

The consequences of Theorem 2 clearly motivate the search for a solution concept that can deliver sharper predictions on allocations that will be visited by the process in the long-run. We take one further step in the analysis of stability and accessibility of allocations. In the following subsection, we analyze the limit invariant distribution which captures not only the number of mistakes needed to switch between recurrent classes but also accounts for details of stability and accessibility of an allocation such as the number of coalitions that can agree or decide to improve upon it.

\subsection{The Limit Invariant Distribution}

So far we have offered a method to identify the set of stochastically stable allocations $S R C(R)$, i.e., the support of the limit invariant distribution $\mu^{*}=\lim _{\epsilon \rightarrow 0} \mu_{\epsilon}$. To assess the actual probability $\mu^{*}(x)$ that the p.d.r. process will be at allocation $x$ in the long run and for vanishing probability of mistakes, the next theorem presents a closed form representation of $\mu^{*}$ that makes explicit use of the polynomial structure of $M^{\epsilon}\left(x^{\prime}, x^{\prime \prime}\right)=\sum_{k=0, \ldots, n} m_{k}\left(x^{\prime}, x^{\prime \prime}\right) \epsilon^{k}$. For further reference we define a least resistance transition $\widetilde{m}\left(x^{\prime}, x^{\prime \prime}\right)$ as the component $m_{k}\left(x^{\prime}, x^{\prime \prime}\right)>0$ that minimizes $k$ (i.e., for any two allocations $x^{\prime}$ and $x^{\prime \prime}, \widetilde{m}\left(x^{\prime}, x^{\prime \prime}\right)$ depicts the respective transition probability with the smallest possible number of mistakes).

Theorem 3. Let $R \in \mathcal{R}^{N}$ and $\left(X, M^{\epsilon}(R)\right)$ be a p.d.r. process. Then, $\mu^{*}(x)=\frac{p(x)}{\sum_{y \in X} p(y)}$ with $p(x)=\sum_{\left\{T \in \mathcal{T}_{x} \mid \sum_{\left[x^{\prime}, x^{\prime \prime}\right] \in T} r\left(x^{\prime}, x^{\prime \prime}\right)=\gamma(x)\right\}}\left[\Pi_{\left[x^{\prime}, x^{\prime \prime}\right] \in T} \widetilde{m}\left(x^{\prime}, x^{\prime \prime}\right)\right]$.

Proof. Freidlin and Wentzell (1998, Chapter 6, Lemma 3.1) show that the (unique) invariant distribution of an irreducible Markov process $(X, M)$ is given by $\mu(x)=\frac{q(x)}{\sum_{y \in X} q(y)}$ with $q(x)=\sum_{T \in \mathcal{T}_{x}} \Pi_{\left[x^{\prime}, x^{\prime \prime}\right] \in T} M\left(x^{\prime}, x^{\prime \prime}\right)$. If the Markov process is a p.d.r. process $\left(X, M^{\epsilon}(R)\right)$ such that $M^{\epsilon}\left(x^{\prime}, x^{\prime \prime}\right)=\sum_{k=0, \ldots, n} m_{k}\left(x^{\prime}, x^{\prime \prime}\right) \epsilon^{k}, q(x)$ is a polynomial of degree $n(|X|-1)$ in the mistake probability $\epsilon$ (there can be only one mistake per agent per edge, and there are $(|X|-1)$ edges in any $x$-tree), i.e., we can rewrite $q(x) \equiv \sum_{k=0, \ldots, n(|X|-1)} q_{k}(x) \epsilon^{k}$. 
By Theorem 2(ii), $\lim _{\epsilon \rightarrow 0} q(x)>0$ if and only if $x \in R C(R)$. Moreover, for all $k<\gamma(x)$, $q_{k}(x)=0$ and $q_{\gamma(x)}(x)>0$ (by the definition of the stochastic potential, there are no $x$-trees with less than $\gamma(x)$ mistakes).

Finally, observe that $q_{\gamma(x)}=\sum_{\left\{T \in \mathcal{T}_{x} \mid \sum_{\left[x^{\prime}, x^{\prime \prime}\right] \in T} r\left(x^{\prime}, x^{\prime \prime}\right)=\gamma(x)\right\}} \Pi_{\left[x^{\prime}, x^{\prime \prime}\right] \in T} \widetilde{m}\left(x^{\prime}, x^{\prime \prime}\right)$ - a contribution to $q(x)$ of lowest order in $\epsilon$ is given by the sum over least resistance trees.

Let $|\mathcal{R C}(R)|=1$. Then, for all $x \in R C(R), \lim _{\epsilon \rightarrow 0} q(x)=q_{0}(x)>0$ and $\mu^{*}(x)=$ $\lim _{\epsilon \rightarrow 0} \mu^{\epsilon}(x)=\frac{q_{0}(x)}{\sum_{y \in R C(R)} q_{0}(y)}$ with $q_{0}(x)=\sum_{\left\{T \in \mathcal{T}_{x} \mid \Pi_{\left[x^{\prime}, x^{\prime \prime}\right] \in T} r\left(x^{\prime}, x^{\prime \prime}\right)=0\right\}} \Pi_{\left[x^{\prime}, x^{\prime \prime}\right] \in T} m_{0}\left(x^{\prime}, x^{\prime \prime}\right)$. Since $\gamma(x)=0, q_{0}(x)=p(x)$.

Let $|\mathcal{R C}(R)| \geq 2$. Recall that for all $x \in X$ and $k<\gamma(x), q_{k}(x)=0$, and for all $x \in R C(R), q_{\gamma(x)}(x)>0$. Then, for all $x \in X$ and $j \in\{0, \ldots,(\gamma(x)-1)\}, \lim _{\epsilon \rightarrow 0} \frac{\partial^{j} q(x)}{\partial \epsilon^{j}}=0$. Moreover, $\lim _{\epsilon \rightarrow 0} \frac{\partial^{\gamma(x)} q(x)}{\partial \epsilon^{\gamma(x)}}=(\gamma(x) !) q_{\gamma(x)}(x)$. Then, iterated application of l'Hospital's rule implies

$$
\mu^{*}(x)=\lim _{\epsilon \rightarrow 0} \mu_{\epsilon}(x)=\lim _{\epsilon \rightarrow 0} \frac{q(x)}{\sum_{y \in X} q(y)}=\lim _{\epsilon \rightarrow 0} \frac{\frac{\partial^{\gamma(x)} q(x)}{\partial \epsilon}(x)}{\sum_{y \in X} \frac{\partial^{\gamma(x)}(x)}{\partial \epsilon \gamma(x)}}=\frac{q_{\gamma(x)}(x)}{\sum_{y \in R C(R)} q_{\gamma(x)}(y)} .
$$

Computation Theorem 3 offers the following procedure to compute $\mu^{*}(x)$ for an allocation $x \in R C(R)$.

1. Construct all least-resistance $x$-trees (i.e., determine $\left\{T \in \mathcal{T}_{x} \mid \sum_{\left[x^{\prime}, x^{\prime \prime}\right] \in T} r\left(x^{\prime}, x^{\prime \prime}\right)=\right.$ $\gamma(x)\})$. By Theorem 2(i), this amounts to a construction of all trees of resistance $|\mathcal{R C}(R)|-1$. We proceed in three steps:

(a) List all recurrent classes $A \in \mathcal{R C}(R)$ and determine all edges from elements of $A$ to elements of $X \backslash A$ with one mistake. ${ }^{13}$

(b) List all edges with zero mistakes.

(c) Compose all $x$-trees with edges listed under (a) and (b).

2. Compute the product of transition probabilities of all edges in a given least-resistance $x$-tree $T$, i.e., $\Pi_{\left[x^{\prime}, x^{\prime \prime}\right] \in T} \widetilde{m}\left(x^{\prime}, x^{\prime \prime}\right)$.

3. Sum over all least resistance $x$-trees.

We illustrate the method by revisiting some of our examples ${ }^{14}$ (for each example, we recapitulate the complete set of preferences and individual rational allocations to help the reader follow the reasoning in the construction of the limit invariant distribution). Apart from the structural results described in our theorems, our analysis of the examples forms an integral part of the paper.

We start with Example 4 to show that the limit invariant distribution serves as a selection device among core (and Walrasian) allocations.

\section{Example 4 (continued). Multiple Walrasian Allocations Recall}

$R_{1}:(1,2),(3,3),(2,3),(1,1)$, anything,

$R_{2}:(1,3),(1,2),(3,3),(2,2)$, anything,

\footnotetext{
${ }^{13}$ By construction, any edge from an element of $A$ to $X \backslash A$ needs at least one mistake. By Theorem 2(i), any such edge in a least resistance tree must not have more than one mistake.

${ }^{14}$ For each example, we recapitulate the complete set of preferences and individual rational allocations to help the reader follow the reasoning in the construction of the limit invariant distribution.
} 
$R_{3}:(2,1),(3,1),(3,3)$, anything,

$\operatorname{IR}(R)=\left\{x^{1}, x^{2}, x^{3}, x^{4}\right\}$ with

$$
\begin{aligned}
& x^{1}=\{(1,1),(2,2),(3,3)\}, \quad x^{2}=\{(2,3),(1,2),(3,1)\}, \quad x^{3}=\{(3,3),(1,2),(2,1)\}, \\
& x^{4}=\{(1,2),(3,3),(2,1)\},
\end{aligned}
$$

and $R C(R)=S R C(R)=$ Core $(R)=W(R)=\left\{x^{3}, x^{4}\right\}$.

Construction of Least Resistance Trees: By Theorem 2, $\gamma\left(x^{3}\right)=\gamma\left(x^{4}\right)=1$.

(a) $\mathcal{R C}(R)=\left\{\left\{x^{3}\right\},\left\{x^{4}\right\}\right\}$. There are two types of edges from $x^{3}$ to $X \backslash\left\{x^{3}\right\}$. Either $x^{4}$ $\{1,2,3\}$-blocks $x^{3}$ (with a mistake by agent 2 ) or a singleton coalition blocks by mistake via $x^{1}$. Similarly, there are two types of edges from $x^{4}$ to $X \backslash\left\{x^{4}\right\}$. Either $x^{3}\{1,2,3\}$ blocks $x^{4}$ (with a mistake by agent 1 ) or a singleton coalition blocks by mistake via $x^{1}$.

(b) The only edges with zero mistakes are from $x^{1}$ to any other individually rational allocation and from $x^{2}$ to $x^{3}$ (all through an agreement of the grand coalition).

(c) Figure 1 depicts the three different types of $x^{3}$-least resistance trees. Two more trees can be constructed by connecting $x^{1}$ with $x^{2}$ or $x^{4}$, respectively, instead of connecting it with $x^{3}$ in the left tree. Figure 2 depicts the two different types of $x^{4}$-trees. Two more trees can be constructed by connecting $x^{1}$ with $x^{2}$ or $x^{3}$, respectively, instead of connecting it with $x^{4}$ in the left tree. Because $x^{3}$ can $\{1,2,3\}$-block $x^{2}$ with no mistake, but $x^{4}$ cannot, there is no $x^{4}$-analogue to the middle $x^{3}$-tree in Figure 1.
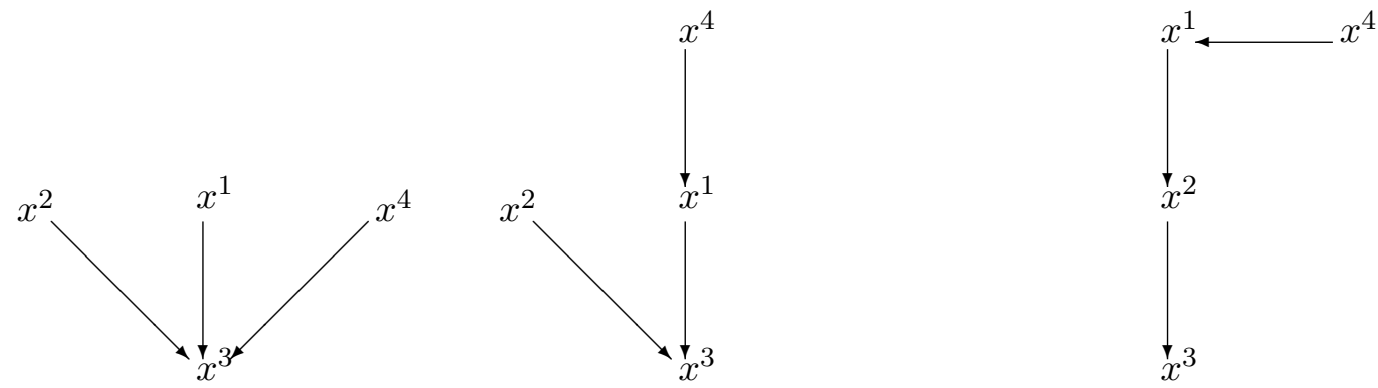

Figure 1: Least Resistance $x^{3}$-trees in Example 4

Computation of the Limit Invariant Distribution: By Theorem 3, and

$$
\frac{\mu^{*}\left(x^{3}\right)}{\widetilde{m}\left(x^{2}, x^{3}\right)}=\widetilde{m}\left(x^{4}, x^{3}\right)\left(\widetilde{m}\left(x^{1}, x^{2}\right)+\widetilde{m}\left(x^{1}, x^{3}\right)+\widetilde{m}\left(x^{1}, x^{4}\right)\right)+\widetilde{m}\left(x^{4}, x^{1}\right)\left(\widetilde{m}\left(x^{1}, x^{3}\right)+\widetilde{m}\left(x^{1}, x^{2}\right)\right) \text {, }
$$

$\frac{\mu^{*}\left(x^{4}\right)}{\tilde{m}\left(x^{2}, x^{3}\right)}=\widetilde{m}\left(x^{3}, x^{4}\right)\left(\widetilde{m}\left(x^{1}, x^{2}\right)+\widetilde{m}\left(x^{1}, x^{3}\right)+\widetilde{m}\left(x^{1}, x^{4}\right)\right)+\widetilde{m}\left(x^{3}, x^{1}\right) \widetilde{m}\left(x^{1}, x^{4}\right)$.

By Assumption 4, the probability to make a mistake does not depend on the identity of the agent. Now suppose that the probability for a certain coalition to form does not depend on the current allocation. Then, $\widetilde{m}\left(x^{3}, x^{4}\right)=\widetilde{m}\left(x^{4}, x^{3}\right)$ and $\widetilde{m}\left(x^{3}, x^{1}\right)=\widetilde{m}\left(x^{4}, x^{1}\right)$. Finally, suppose that $\widetilde{m}\left(x^{1}, x^{3}\right)=\widetilde{m}\left(x^{1}, x^{4}\right)$ (i.e., the grand coalition agrees upon $x^{3}$ and $x^{4}$ if the p.d.r. process is in $x^{1}$ with the same probability). Then, $\mu^{*}\left(x^{3}\right)>\mu^{*}\left(x^{4}\right)$ and the process is more likely to be found at allocation $x^{3}$ then at $x^{4}$ in the long-run. 

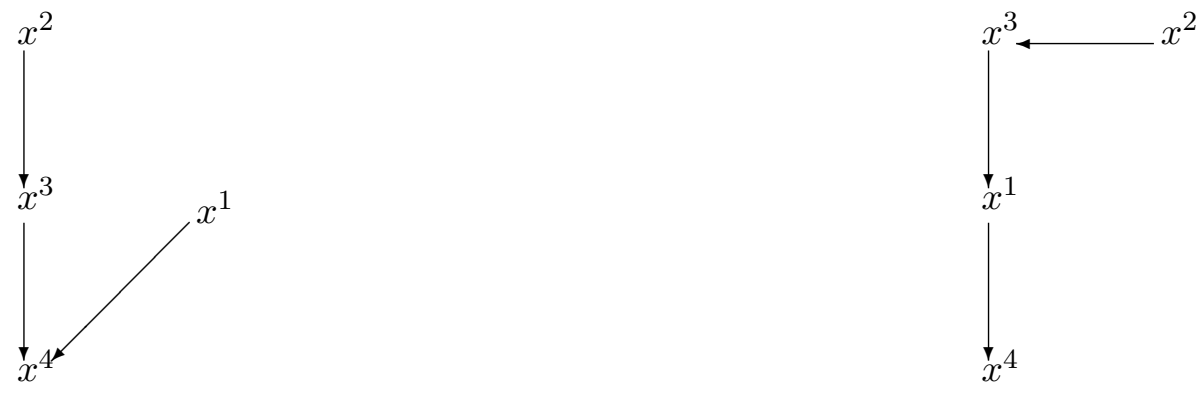

Figure 2: Least Resistance $x^{4}$-trees in Example 4

Observe that this result is driven by the fact that $x^{3}$ can $\{1,2,3\}$-block $x^{2}$ without mistake while $x^{4}$ cannot. This difference in mistake-free edges leads to a larger set of least resistance $x^{3}$-trees and thereby enhances the respective component in the limit invariant distribution. $\diamond$

This example shows that the various core (Walrasian) allocations of a multiple-type housing market are not necessarily final allocations of the d.r. process with the same probability. Accordingly, one may wonder wether Walrasian allocations are more likely final allocations than non-Walrasian core allocations, and wether core allocations are more likely final allocations than non-core recurrent allocations. To elaborate on the first issue, we reconsider Example 3.

Example 3 (continued). $S R C(R)=R C(R)=\operatorname{Core}(R) \supsetneq W(R)$ Recall

$R_{1}:(1,2),(3,3),(2,3),(1,1)$, anything,

$R_{2}:(1,3),(1,2),(3,3),(2,2)$, anything,

$R_{3}:(3,1),(2,1),(3,3)$, anything,

$\operatorname{IR}(R)=\left\{x^{1}, x^{2}, x^{3}, x^{4}\right\}$ with

$x^{1}=\{(1,1),(2,2),(3,3)\}, \quad x^{2}=\{(2,3),(1,2),(3,1)\}, \quad x^{3}=\{(1,2),(3,3),(2,1)\}$,

$x^{4}=\{(3,3),(1,2),(2,1)\}$,

and $\left\{x^{2}, x^{3}, x^{4}\right\}=R C(R)=S R C(R)=\operatorname{Core}(R) \supsetneq W(R)=\left\{x^{3}\right\}$.

Observe that a direct edge from $x^{2}$ to $x^{3}$ takes two mistakes while all other transitions from one core allocation to another need only one mistake. This suggests that $x^{2}$ is less likely to be left than $x^{3}$ and $x^{4}$ (i.e., it is more stable), while $x^{3}$ is less likely to be reached by the p.d.r. process than $x^{2}$ and $x^{4}$ (i.e., it is less accessible). In Appendix A we prove that this intuition is true and indeed $\mu^{*}\left(x^{2}\right)>\mu^{*}\left(x^{4}\right)>\mu^{*}\left(x^{3}\right)$ if the probability that a certain coalition becomes active does not depend on the allocation and a given coalition agrees upon each improvement with the same probability. Hence, $x^{2}$ is the most accessible and stable allocation while $x^{3}$, the (unique) Walrasian allocation, is the worst prediction - among all recurrent allocations - for the long-run behavior of the d.r. process.

Finally, we demonstrate by the following example that cycles of individually rational (noncore) allocations can be more likely final allocations of the d.r. process than core allocations. 
Example 2 (continued). $S R C(R)=R C(R) \nsupseteq$ Core $(R)$ Recall

$R_{1}:(3,1),(2,2),(1,2),(1,1)$, anything,

$R_{2}:(2,1),(3,3),(3,2),(2,2)$, anything,

$R_{3}:(2,3),(1,1),(1,3),(3,3)$, anything,

$\operatorname{IR}(R)=\left\{x^{1}, x^{2}, x^{3}, x^{4}, x^{5}\right\}$ with,

$x^{1}=\{(1,1),(2,2),(3,3)\}, \quad x^{2}=\{(1,1),(3,2),(2,3)\}, \quad x^{3}=\{(1,2),(2,1),(3,3)\}$,

$x^{4}=\{(3,1),(2,2),(1,3)\}, \quad x^{5}=\{(2,2),(3,3),(1,1)\}$,

and $\operatorname{Core}(R)=W(R)=\left\{x^{5}\right\}$ and $R C(R)=S R C(R)=\left\{x^{2}, x^{3}, x^{4}, x^{5}\right\}$.

Construction of Least Resistance Trees: By Theorem 2, $\gamma\left(x^{2}\right)=\gamma\left(x^{3}\right)=\gamma\left(x^{4}\right)=\gamma\left(x^{5}\right)=1$.

(a) $\mathcal{R C}(R)=\left\{\left\{x^{2}, x^{3}, x^{4}\right\},\left\{x^{5}\right\}\right\}$. There are two types of edges from an element of $\left\{x^{2}, x^{3}, x^{4}\right\}$ to $X \backslash\left\{x^{2}, x^{3}, x^{4}\right\}$. Either, $x^{5}\{1,2,3\}$-blocks an element of $\left\{x^{2}, x^{3}, x^{4}\right\}$ with one agent making a mistake or $x^{1}$ blocks an element of $\left\{x^{2}, x^{3}, x^{4}\right\}$ with a singleton coalition making a mistake. Similarly, there are two types of edges from $x^{5}$ to an element of $X \backslash\left\{x^{5}\right\}$. Either an element of $\left\{x^{2}, x^{3}, x^{4}\right\}$ blocks $x^{5}$ via a coalition of two agents with one agent making a mistake (e.g., $x^{2}\{2,3\}$-blocks $x^{5}$ if agent 2 makes a mistake) or $x^{1}$ blocks $x^{5}$ with a singleton coalition making a mistake.

(b) The only edges with zero mistakes are from $x^{1}$ to $x^{5}$ (through an agreement of the grand coalition) and from $x^{1}$ to any element of $\left\{x^{2}, x^{3}, x^{4}\right\}$ (through an agreement of the grand coalition or a particular coalition of two agents, e.g., $x^{2}\{2,3\}$-blocks $\left.x^{1}\right)$.

(c) Figure 3 depicts the two different types of $x^{5}$-least resistance trees. In total, there are 12 trees of Type (a) and 3 trees of Type (b) (to see this consider the different permutations of elements of $\left\{x^{2}, x^{3}, x^{4}\right\}$ and the different edges to connect $\left.x^{1}\right)$. Figure 4 depicts the two different types of $x^{2}$-trees $\left(x^{3}\right.$ and $x^{4}$-trees look identical and only differ in the respective active coalitions). In total, there are 12 trees of Type (a) and 3 trees of Type (b). A complete list of least resistance trees can be found in Appendix A).

Computation of the Limit Invariant Distribution: In contrast to Example 4 (see above), the number of least resistance $x^{2}$ - and $x^{5}$-trees is identical. However, the respective edges resemble different transition probabilities. In the following, we present an informal discussion of the relevant effects - a complete treatment is relegated to Appendix A. To start out, observe that $x^{2}$ - and $x^{5}$-trees differ in three respects. First, any least resistance $x^{5}$-tree of Type (a) in Figure 3 contains a blocking of a cycle allocation by the grand coalition to reach $x^{5}$, while $x^{2}$-trees of Type (a) in Figure 4 need a respective blocking of $x^{5}$ by a coalition of two agents. If the probability that the grand coalition is chosen equals the probability that any coalition with two agents is chosen, this difference does not matter. Second, any least resistance $x^{5}$-tree of Type (b) in Figure 3 contains a blocking of a cycle allocation by a singleton coalition to reach $x^{1}$ (by mistake), while $x^{2}$-trees of Type (b) in Figure 4 need a respective blocking of $x^{5}$. However, in every cycle allocation one of the agents is already at his endowment such that the process cannot transit to $x^{1}$ with this agent making a mistake. Hence, $\widetilde{m}\left(x^{5}, x^{1}\right)>\widetilde{m}\left(x^{2}, x^{1}\right)$ if the probability that a certain singleton coalition forms does not depend on the allocation. This leads to a larger contribution from this type of trees to $\mu^{*}\left(x^{2}\right)$ than to $\mu^{*}\left(x^{5}\right)$. Third, $x^{5}$-trees of type (b) in Figure 3 contain a blocking of $x^{1}$ by 

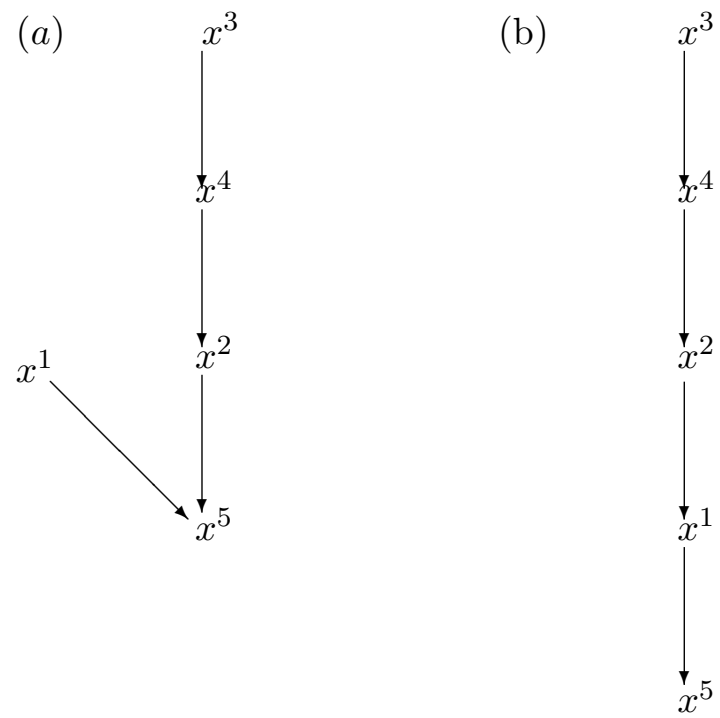

Figure 3: Least Resistance $x^{5}$-trees
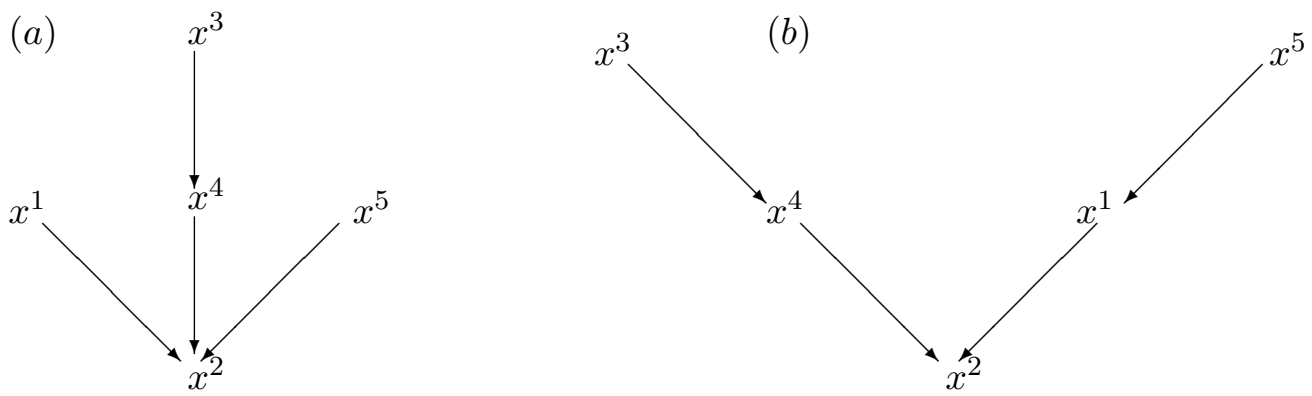

Figure 4: Least Resistance $x^{2}$-trees

$x^{5}$, while $x^{2}$-trees of type (b) in Figure 4 contain a blocking of $x^{1}$ by $x^{2}$. While the former blocking can only be agreed upon by the grand coalition, the latter can also be achieved by $\{2,3\}$. Hence, $\widetilde{m}\left(x^{1}, x^{2}\right)>\widetilde{m}\left(x^{1}, x^{5}\right)$ if a coalition agrees upon every blocking with the same probability. In Appendix A we prove that indeed $\mu^{*}\left(x^{2}\right)>\mu^{*}\left(x^{5}\right)$ if $\widetilde{m}\left(x, x^{1}\right)<\widetilde{m}\left(x^{5}, x^{1}\right)$ for every $x \in\left\{x^{2}, x^{3}, x^{4}\right\}, \widetilde{m}\left(x^{1}, x^{2}\right)>\widetilde{m}\left(x^{1}, x^{5}\right)$, and coalitions of size three form with the same probability as coalitions of size two. Hence, the probability that the process is found in one cycle allocation is higher than the respective probability to find it in the core. ${ }^{15}$ Note, however, that this result is not driven by the lack of mistake-free edges that lead to the core allocation $x^{5}$ (as in the previous examples), but by details of the process that determine the opportunities to reach and exit the different recurrent classes.

The above-mentioned examples highlight the following features of the limit invariant distribution. First, the probability that a dynamic recontracting process is in a certain recurrent class does not depend on its topology (singleton or cycle). Neither is it necessarily enhanced by the fact that a certain allocation is in the core or Walrasian. The value of the limit invariant distribution rather depends on two issues: (i) The accessibility of a recurrent class

\footnotetext{
${ }^{15}$ In Appendix A we show that symmetry implies $\mu^{*}\left(x^{2}\right)=\mu^{*}\left(x^{3}\right)=\mu^{*}\left(x^{4}\right)$ under these conditions.
} 
from individually rational allocations that are not member of a recurrent class. An example is allocation $x^{2}$ in Example 4. (ii) The number and relative transition probabilities of resistance minimizing paths from one recurrent class to another. Consider as an example the importance of the edges to and from the endowment in Example 2.

\section{Concluding Remarks}

Recurrent Classes We first model economic interaction in multiple-type housing markets by an unperturbed dynamic recontracting process. Then, an obvious solution concept for any given multiple-type housing market $R$ is its set of recurrent classes $\mathcal{R C}(R)$ and the set of recurrent allocations $R C(R)$. The set of recurrent classes of the dynamic recontracting process is non-empty and contains all sets of allocations that cannot be blocked by any allocation outside the respective recurrent class. Every element of the core forms a singleton recurrent class. Moreover, non-singleton recurrent classes exist (in settings with an empty core as well as vis-a-vis a non-empty core).

Stochastically Stable Allocations Next, we allow agents to make mistakes and model economic interaction in multiple-type housing markets by a perturbed dynamic recontracting process. The second non-empty solution concepts for any given multiple-type housing market $R$ then is its set of stochastically stable allocations $S R C(R)$. If the perturbation of the dynamic recontracting process through mistakes becomes sufficiently small (by letting mistake probabilities converge to zero), the process will - in the long-run - converge to a non-empty subset of the set of recurrent allocations (the set of stochastically stable allocations). We show that every recurrent class is stochastically stable.

Limit Invariant Distributions Finally, to better understand which allocations are likely to be final allocations of our dynamic process of trade, we introduced a method to directly access the limit invariant distribution. By construction, the limit invariant distribution $\mu^{*}$ of a dynamic recontracting process is the unique probability distribution over allocations that the dynamic recontracting process will converge to in the long-run. By considering the complete set of least resistance trees in the computation of the limit invariant distribution, the interplay between stability and accessibility that determines the final allocation is better captured than by a stochastic stability analysis (as illustrated in several examples). However, the extra information obtained clearly comes at the cost of having to compute all least resistance trees (compared to the construction of one tree for a stochastic stability analysis). Since any least resistance tree only consists of resistance-minimizing edges, this boils down to a simple combinatorial exercise (as also demonstrated in several examples).

Calculating the Limit Invariant Distribution Apart from our analysis of dynamic recontracting processes for multiple-type housing markets, our paper also aims at an illustration of the method to compute the limit invariant distribution. The particular features of multiple-type housing markets proved useful to demonstrate its applicability and intuitive appeal. However, results similar to Theorem 3 are also feasible and desirable for other Markov processes, for instance for non-cooperative games or social and economic network formation. 
Conclusion Our analysis has shown that neither the core nor Walrasian allocations might be good predictors for dynamic recontracting processes. We have seen some instances where a cycle of allocations may be the best predictor for the final allocation of our dynamic trading process. Clearly, this conclusion is in part driven by the myopia of agents. Recall that in each period agents agree to trade if it is beneficial to do so. In accepting such trades, agents do not envision the possible blockings along the trading path. Nevertheless, it is clear from our analysis that there are aspects of stability and in particular of accessibility of allocations that are not captured by the core. Some core allocations turn out to be harder to reach than others while non-core allocations may emerge naturally through a sequence of trades. This indicates that modeling the dynamics of recontracting only implicitly by a cooperative solution concept as the core is inappropriate (even though we have modeled our dynamic recontracting process with a core bias by never transferring property rights).

\section{Appendix}

\section{A Examples}

\section{Example 1: An Empty Core Recall}

$R_{1}:(3,1),(1,2),(1,1)$, anything,

$R_{2}:(2,1),(3,2),(2,2)$, anything,

$R_{3}:(2,3),(1,3),(3,3)$, anything,

and $\operatorname{IR}(R)=\left\{x^{1}, x^{2}, x^{3}, x^{4}\right\}$ with

$$
\begin{aligned}
& x^{1}=\{(1,1),(2,2),(3,3)\}, \quad x^{2}=\{(1,1),(3,2),(2,3)\}, \quad x^{3}=\{(1,2),(2,1),(3,3)\}, \\
& x^{4}=\{(3,1),(2,2),(1,3)\} .
\end{aligned}
$$

Hence, $\operatorname{Core}(R)=W(R)=\emptyset$. Furthermore, $R C(R)=S R C(R)=\left\{x^{2}, x^{3}, x^{4}\right\}$.

Example 2: The Unique Walrasian Allocation Equals the Core Allocation Recall

$R_{1}:(3,1),(2,2),(1,2),(1,1)$, anything,

$R_{2}:(2,1),(3,3),(3,2),(2,2)$, anything,

$R_{3}:(2,3),(1,1),(1,3),(3,3)$, anything,

and $\operatorname{IR}(R)=\left\{x^{1}, x^{2}, x^{3}, x^{4}, x^{5}\right\}$ with

$$
\begin{array}{ll}
x^{1}=\{(1,1),(2,2),(3,3)\}, & x^{2}=\{(1,1),(3,2),(2,3)\}, \quad x^{3}=\{(1,2),(2,1),(3,3)\}, \\
x^{4}=\{(3,1),(2,2),(1,3)\}, & x^{5}=\{(2,2),(3,3),(1,1)\} .
\end{array}
$$

Clearly, $x^{2}\{2,3\}$-blocks $x^{1}, x^{3}\{1,2\}$-blocks $x^{2}, x^{4}\{1,3\}$-blocks $x^{3}$, and $x^{2}\{2,3\}$-blocks $x^{4}$. Since $x^{5}$ cannot be blocked by any coalition, $\operatorname{Core}(R)=\left\{x^{5}\right\}$. Next, we prove that allocation $x^{5}$ is Walrasian. It is easy to check that the following (in)equalities hold, e.g., for price system $p \equiv\left(p_{1}, p_{2}\right)$ such that $p_{1}=\left(0,1, \frac{1}{2}\right)$ and $p_{2}=\left(1,0, \frac{1}{2}\right)$ : 
(1) $p_{1}(1)+p_{2}(1)=p_{1}(2)+p_{2}(2), \quad(2) p_{1}(2)+p_{2}(2)=p_{1}(3)+p_{2}(3)$,

(3) $p_{1}(3)+p_{2}(3)=p_{1}(1)+p_{2}(1), \quad$ (4) $p_{1}(3)+p_{2}(1)>p_{1}(1)+p_{2}(1)$,

(5) $p_{1}(2)+p_{2}(1)>p_{1}(2)+p_{2}(2), \quad(6) p_{1}(2)+p_{2}(3)>p_{1}(3)+p_{2}(3)$.

Hence, $W(R)=\left\{x^{5}\right\}$. Furthermore, $R C(R)=S R C(R)=\left\{x^{2}, x^{3}, x^{4}, x^{5}\right\}$.

To compute the limit invariant distribution, we proceed as indicated in Section 5.2.

Construction of Least Resistance Trees: By Theorem 2, $\gamma\left(x^{5}\right)=\gamma\left(x^{2}\right)=\gamma\left(x^{3}\right)=\gamma\left(x^{4}\right)=1$.

(a) $\mathcal{R C}(R)=\left\{\left\{x^{2}, x^{3}, x^{4}\right\},\left\{x^{5}\right\}\right\}$. The following table lists the coalitions that facilitate a transition from an initial state (row) to a final state (column) with one mistake ${ }^{16}$ (we omit the diagonal elements as they are irrelevant for cycle-free graphs, moreover we do not list edges with mistakes between allocations in $\left\{x^{2}, x^{3}, x^{4}\right\}$ as they can never be part of a least resistance tree).

\begin{tabular}{|c|c|c|c|c|c|}
\hline & $x^{1}$ & $x^{2}$ & $x^{3}$ & $x^{4}$ & $x^{5}$ \\
\hline$x^{1}$ & - & $\emptyset$ & $\emptyset$ & $\emptyset$ & $\emptyset$ \\
\hline$x^{2}$ & $\{1\},\{2\},\{3\},\{1,2\},\{1,3\}$ & - & $N$ & - & $N$ \\
\hline$x^{3}$ & $\{1\},\{2\},\{3\},\{1,3\},\{2,3\}$ & - & - & $N$ & $N$ \\
\hline$x^{4}$ & $\{1\},\{2\},\{3\},\{1,2\},\{2,3\}$ & $N$ & - & - & $N$ \\
\hline$x^{5}$ & $\{1\},\{2\},\{3\}$ & $\{2,3\}$ & $\{1,2\}$ & $\{1,3\}$ & - \\
\hline
\end{tabular}

(b) The following table lists the coalitions that facilitate a transition from an initial state (row) to a final state (column) with zero mistakes.

\begin{tabular}{|c|c|c|c|c|c|}
\hline & $x^{1}$ & $x^{2}$ & $x^{3}$ & $x^{4}$ & $x^{5}$ \\
\hline$x^{1}$ & $\mathrm{x}$ & $N,\{2,3\}$ & $N,\{1,2\}$ & $N,\{1,3\}$ & $N$ \\
\hline$x^{2}$ & $\emptyset$ & - & $\{1,2\}$ & $\emptyset$ & $\emptyset$ \\
\hline$x^{3}$ & $\emptyset$ & $\emptyset$ & - & $\{1,3\}$ & $\emptyset$ \\
\hline$x^{4}$ & $\emptyset$ & $\{2,3\}$ & $\emptyset$ & - & $\emptyset$ \\
\hline$x^{5}$ & $\emptyset$ & $\emptyset$ & $\emptyset$ & $\emptyset$ & - \\
\hline
\end{tabular}

(c) With the transition opportunities as depicted in the previous tables, we can now list all least resistance trees for the (unique) core allocation $x^{5}$ and a cycle allocation, for instance $x^{2}$. We start with the set of $x^{5}$-trees. If every least resistance tree includes exactly one mistake (i.e., $\gamma\left(x^{5}\right)=1$ ), this mistake has to be made on a (cycle-free) sequence of edges from an element of the cycle to $x^{5}$. From any allocation in the cycle there are exactly two such paths. First, the grand coalition forms and the agent who is worse off at allocation $x^{5}$ (note that this is exactly one agent for every allocation in the cycle) agrees upon $x^{5}$ by mistake. Second, a singleton coalition forms and the respective agent ask for his endowment even though he is then worse off. Note that in every cycle allocation there are two such singleton allocations (e.g., at $x^{2}$ only agents 2 and 3 can recontract on their endowment and thereby induce a transition to $x^{1}$. Agent 1 , in contrast, is already at his endowment). These two types of paths induce the two types of least resistance trees depicted in Figure 3. Next, observe that the allocations of the cycle have to be connected via the respective (unique) mistake-free edges. As for

\footnotetext{
${ }^{16}$ The agent who makes a mistake is uniquely determined in this example.
} 
type (a) note that there are three different allocations from which the cycle can be left to $x^{5}$. Moreover, there can be a (mistake-free) edge from $x^{1}$ to any other of the four allocations. This leaves us with the following 12 trees of type (a).

$$
\begin{aligned}
& \left\{\left[x^{3}, x^{4}\right],\left[x^{4}, x^{2}\right],\left[x^{2}, x^{5}\right],\left[x^{1}, x^{2}\right]\right\} ;\left\{\left[x^{3}, x^{4}\right],\left[x^{4}, x^{2}\right],\left[x^{2}, x^{5}\right],\left[x^{1}, x^{3}\right]\right\} ; \\
& \left\{\left[x^{3}, x^{4}\right],\left[x^{4}, x^{2}\right],\left[x^{2}, x^{5}\right],\left[x^{1}, x^{4}\right]\right\} ;\left\{\left[x^{3}, x^{4}\right],\left[x^{4}, x^{2}\right],\left[x^{2}, x^{5}\right],\left[x^{1}, x^{5}\right]\right\} ; \\
& \left\{\left[x^{4}, x^{2}\right],\left[x^{2}, x^{3}\right],\left[x^{3}, x^{5}\right],\left[x^{1}, x^{2}\right]\right\} ;\left\{\left[x^{4}, x^{2}\right],\left[x^{2}, x^{3}\right],\left[x^{3}, x^{5}\right],\left[x^{1}, x^{3}\right]\right\} ; \\
& \left\{\left[x^{4}, x^{2}\right],\left[x^{2}, x^{3}\right],\left[x^{3}, x^{5}\right],\left[x^{1}, x^{4}\right]\right\} ;\left\{\left[x^{4}, x^{2}\right],\left[x^{2}, x^{3}\right],\left[x^{3}, x^{5}\right],\left[x^{1}, x^{5}\right]\right\} ; \\
& \left\{\left[x^{2}, x^{3}\right],\left[x^{3}, x^{4}\right],\left[x^{4}, x^{5}\right],\left[x^{1}, x^{2}\right]\right\} ;\left\{\left[x^{2}, x^{3}\right],\left[x^{3}, x^{4}\right],\left[x^{4}, x^{5}\right],\left[x^{1}, x^{3}\right]\right\} ; \\
& \left\{\left[x^{2}, x^{3}\right],\left[x^{3}, x^{4}\right],\left[x^{4}, x^{5}\right],\left[x^{1}, x^{4}\right]\right\} ;\left\{\left[x^{2}, x^{3}\right],\left[x^{3}, x^{4}\right],\left[x^{4}, x^{5}\right],\left[x^{1}, x^{5}\right]\right\} .
\end{aligned}
$$

Likewise there are three different allocations in the cycle from which $x^{1}$ can be reached (and subsequently left towards $x^{5}$ ). Hence, we have to add the following three trees.

$$
\begin{aligned}
& \left\{\left[x^{3}, x^{4}\right],\left[x^{4}, x^{2}\right],\left[x^{2}, x^{1}\right],\left[x^{1}, x^{5}\right]\right\} ;\left\{\left[x^{2}, x^{3}\right],\left[x^{3}, x^{4}\right],\left[x^{4}, x^{1}\right],\left[x^{1}, x^{5}\right]\right\} ; \\
& \left\{\left[x^{4}, x^{2}\right],\left[x^{2}, x^{3}\right],\left[x^{3}, x^{1}\right],\left[x^{1}, x^{5}\right]\right\} .
\end{aligned}
$$

In a similar way, we can construct the set of $x^{2}$-trees. If every least resistance tree includes exactly one mistake (i.e., $\gamma\left(x^{2}\right)=1$ ), this mistake has to be made on a (cyclefree) sequence of edges from $x^{5}$ to an element of the cycle. To any allocation in the cycle there are two such paths. Either, a particular coalition of size two forms and the agent who is worse off at the cycle-allocation agrees upon the cycle allocation by mistake. Note that there is exactly one coalition of size two that can actually contract upon each cycle allocation (the agent who is left at his endowment can not be a member of such a coalition). Second, one of the three singleton coalition forms and the respective agent asks for his endowment even though he is then worse off, afterwards the grand coalition or $\{2,3\}$ agree upon $x^{2}$. These two types of paths induce the two types of least resistance trees depicted in Figure 4 (again the allocations of the cycle have to be connected via the respective (unique) mistake-free edges). As for type (a) note that there are three different allocations from which the cycle can be accessed from $x^{5}$. Moreover, there can be a (mistake-free) edge from $x^{1}$ to any other of the four allocation. This leaves us with the following $12 x^{2}$-trees of type (a).

$$
\begin{aligned}
& \left\{\left[x^{3}, x^{4}\right],\left[x^{4}, x^{2}\right],\left[x^{5}, x^{2}\right],\left[x^{1}, x^{2}\right]\right\} ;\left\{\left[x^{3}, x^{4}\right],\left[x^{4}, x^{2}\right],\left[x^{5}, x^{2}\right],\left[x^{1}, x^{3}\right]\right\} ; \\
& \left\{\left[x^{3}, x^{4}\right],\left[x^{4}, x^{2}\right],\left[x^{5}, x^{2}\right],\left[x^{1}, x^{4}\right]\right\} ;\left\{\left[x^{3}, x^{4}\right],\left[x^{4}, x^{2}\right],\left[x^{5}, x^{2}\right],\left[x^{1}, x^{5}\right]\right\} ; \\
& \left\{\left[x^{4}, x^{2}\right],\left[x^{2}, x^{3}\right],\left[x^{5}, x^{3}\right],\left[x^{1}, x^{2}\right]\right\} ;\left\{\left[x^{4}, x^{2}\right],\left[x^{2}, x^{3}\right],\left[x^{5}, x^{3}\right],\left[x^{1}, x^{3}\right]\right\} ; \\
& \left\{\left[x^{4}, x^{2}\right],\left[x^{2}, x^{3}\right],\left[x^{5}, x^{3}\right],\left[x^{1}, x^{4}\right]\right\} ;\left\{\left[x^{4}, x^{2}\right],\left[x^{2}, x^{3}\right],\left[x^{5}, x^{3}\right],\left[x^{1}, x^{5}\right]\right\} ; \\
& \left\{\left[x^{2}, x^{3}\right],\left[x^{3}, x^{4}\right],\left[x^{5}, x^{4}\right],\left[x^{1}, x^{2}\right]\right\} ;\left\{\left[x^{2}, x^{3}\right],\left[x^{3}, x^{4}\right],\left[x^{5}, x^{4}\right],\left[x^{1}, x^{3}\right]\right\} ; \\
& \left\{\left[x^{2}, x^{3}\right],\left[x^{3}, x^{4}\right],\left[x^{5}, x^{4}\right],\left[x^{1}, x^{4}\right]\right\} ;\left\{\left[x^{2}, x^{3}\right],\left[x^{3}, x^{4}\right],\left[x^{5}, x^{4}\right],\left[x^{1}, x^{5}\right]\right\} .
\end{aligned}
$$

Likewise there are three different allocations in the cycle that can be reached from $x^{1}$ (after $x^{5}$ has been left towards $x^{1}$ ). Hence, we have to add the following three trees.

$$
\begin{aligned}
& \left\{\left[x^{3}, x^{4}\right],\left[x^{4}, x^{2}\right],\left[x^{1}, x^{2}\right],\left[x^{5}, x^{1}\right]\right\} ;\left\{\left[x^{2}, x^{3}\right],\left[x^{3}, x^{4}\right],\left[x^{1}, x^{4}\right],\left[x^{5}, x^{1}\right]\right\} ; \\
& \left\{\left[x^{4}, x^{2}\right],\left[x^{2}, x^{3}\right],\left[x^{1}, x^{3}\right],\left[x^{5}, x^{1}\right]\right\} .
\end{aligned}
$$


Computation of the Limit Invariant Distribution: This is sufficient information to apply the formula for $\mu^{*}$ in Theorem 3. For expositional ease let us make the following assumptions. First, recall from Assumption 4 that the probability for an agent to commit a mistake does not depend on his identity, the coalition, or the allocation. Moreover, suppose the probability that a certain coalition forms does not depend on the identity of the agents and that a coalition chooses each improving allocation with the same probability. Then,

$$
\begin{aligned}
& \widetilde{m}\left(x^{2}, x^{3}\right)=\widetilde{m}\left(x^{3}, x^{4}\right)=\widetilde{m}\left(x^{4}, x^{2}\right), \\
& \widetilde{m}\left(x^{1}, x^{2}\right)=\widetilde{m}\left(x^{1}, x^{3}\right)=\widetilde{m}\left(x^{1}, x^{4}\right), \\
& \widetilde{m}\left(x^{2}, x^{1}\right)=\widetilde{m}\left(x^{3}, x^{1}\right)=\widetilde{m}\left(x^{4}, x^{1}\right), \\
& \widetilde{m}\left(x^{2}, x^{5}\right)=\widetilde{m}\left(x^{3}, x^{5}\right)=\widetilde{m}\left(x^{4}, x^{5}\right), \\
& \widetilde{m}\left(x^{5}, x^{2}\right)=\widetilde{m}\left(x^{5}, x^{3}\right)=\widetilde{m}\left(x^{5}, x^{4}\right) .
\end{aligned}
$$

and

$$
\frac{\mu^{*}\left(x^{5}\right)}{\mu^{*}\left(x^{2}\right)}=\frac{9 \widetilde{m}\left(x^{2}, x^{5}\right) \widetilde{m}\left(x^{1}, x^{2}\right)+3 \widetilde{m}\left(x^{1}, x^{5}\right) \widetilde{m}\left(x^{2}, x^{5}\right)+3 \widetilde{m}\left(x^{1}, x^{5}\right) \widetilde{m}\left(x^{2}, x^{1}\right)}{9 \widetilde{m}\left(x^{5}, x^{2}\right) \widetilde{m}\left(x^{1}, x^{2}\right)+3 \widetilde{m}\left(x^{1}, x^{5}\right) \widetilde{m}\left(x^{5}, x^{2}\right)+3 \widetilde{m}\left(x^{5}, x^{1}\right) \widetilde{m}\left(x^{1}, x^{2}\right)}
$$

By checking the relations $\widetilde{m}\left(x^{2}, x^{5}\right) / \widetilde{m}\left(x^{5}, x^{2}\right), \widetilde{m}\left(x^{1}, x^{5}\right) / \widetilde{m}\left(x^{1}, x^{2}\right)$, and $\widetilde{m}\left(x^{2}, x^{1}\right) / \widetilde{m}\left(x^{5}, x^{1}\right)$ we determine the ratio of $\mu^{*}\left(x^{5}\right)$ and $\mu^{*}\left(x^{2}\right)$.

$\widetilde{m}\left(x^{2}, x^{5}\right) / \widetilde{m}\left(x^{5}, x^{2}\right)$ : recall from the second table (see above) that a least resistance edge from $x^{2}$ to $x^{5}$ needs the grand coalition to form and agent 3 making a mistake, while the least resistance edge from $x^{5}$ to $x^{2}$ needs the coalition $\{2,3\}$ to form and agent 2 making a mistake. Suppose coalitions of size two and size three form with the same probability, then $\widetilde{m}\left(x^{2}, x^{5}\right)=\widetilde{m}\left(x^{5}, x^{2}\right)$.

$\widetilde{m}\left(x^{1}, x^{5}\right) / \widetilde{m}\left(x^{1}, x^{2}\right)$ : recall from the first table (see above) that $x^{2}$ can be recontracted upon at $x^{1}$ by the grand coalition and $\{2,3\}$, while the grand coalition is needed to agree upon $x^{5}$. If all improving allocations are chosen from a coalition with the same probability, we therefore get $\widetilde{m}\left(x^{1}, x^{5}\right)<\widetilde{m}\left(x^{1}, x^{2}\right)$.

$\widetilde{m}\left(x^{2}, x^{1}\right) / \widetilde{m}\left(x^{5}, x^{1}\right)$ : the above conditions also ensure that $\widetilde{m}\left(x^{2}, x^{1}\right)<\widetilde{m}\left(x^{5}, x^{1}\right)$.

Hence, $\mu^{*}\left(x^{2}\right)>\mu^{*}\left(x^{5}\right)$. Finally the symmetry of the preferences with respect to a permutation of allocations $x^{2}, x^{3}$, and $x^{4}$ implies that $\mu^{*}\left(x^{2}\right)=\mu^{*}\left(x^{3}\right)=\mu^{*}\left(x^{4}\right)$ whenever coalition formation does not depend on allocations and the identity of the agents.

Example 3: Multiple Core Allocations and a Unique Walrasian Allocation Recall

$R_{1}:(1,2),(3,3),(2,3),(1,1)$, anything,

$R_{2}:(1,3),(1,2),(3,3),(2,2)$, anything,

$R_{3}:(3,1),(2,1),(3,3)$, anything,

and $\operatorname{IR}(R)=\left\{x^{1}, x^{2}, x^{3}, x^{4}\right\}$ with

$$
\begin{aligned}
& x^{1}=\{(1,1),(2,2),(3,3)\}, \quad x^{2}=\{(2,3),(1,2),(3,1)\}, \quad x^{3}=\{(1,2),(3,3),(2,1)\}, \\
& x^{4}=\{(3,3),(1,2),(2,1)\} .
\end{aligned}
$$


Clearly, $x^{2}\{1,2,3\}$-blocks $x^{1}$. Since $x^{2}, x^{3}$, and $x^{4}$ cannot be blocked by any coalition, Core $(R)=\left\{x^{2}, x^{3}, x^{4}\right\}$. Next, we check if any of the core allocations is Walrasian.

Allocation $x^{2}$ is not Walrasian. If it was, then the following (in)equalities would hold:

(1) $p_{1}(1)+p_{2}(1)=p_{1}(2)+p_{2}(3), \quad(2) p_{1}(2)+p_{2}(2)=p_{1}(1)+p_{2}(2)$,

(3) $p_{1}(3)+p_{2}(3)=p_{1}(3)+p_{2}(1), \quad(4) p_{1}(3)+p_{2}(3)>p_{1}(1)+p_{2}(1)$,

(5) $p_{1}(1)+p_{2}(2)>p_{1}(1)+p_{2}(1), \quad(6) p_{1}(1)+p_{2}(3)>p_{1}(2)+p_{2}(2)$.

By $(2)$ and (6), we obtain $p_{1}(1)+p_{2}(3)>p_{1}(1)+p_{2}(2)$. Thus, $p_{2}(3)>p_{2}(2)$. By $(5), p_{2}(2)>$ $p_{2}(1)$ and by $(3), p_{2}(1)=p_{2}(3)$. Hence, $p_{2}(3)>p_{2}(2)>p_{2}(1)=p_{2}(3)$; a contradiction.

Allocation $x^{3}$ is Walrasian. It is easy to check that the following (in)equalities hold, e.g., for price system $p \equiv\left(p_{1}, p_{2}\right)$ such that $p_{1}=\left(2, \frac{1}{2}, 1\right)$ and $p_{2}=\left(1,1, \frac{1}{2}\right)$ :

(1) $p_{1}(1)+p_{2}(1)=p_{1}(1)+p_{2}(2), \quad(2) p_{1}(2)+p_{2}(2)=p_{1}(3)+p_{2}(3)$,

(3) $p_{1}(3)+p_{2}(3)=p_{1}(2)+p_{2}(1), \quad$ (4) $p_{1}(1)+p_{2}(2)>p_{1}(2)+p_{2}(2)$,

(5) $p_{1}(1)+p_{2}(3)>p_{1}(2)+p_{2}(2), \quad(6) p_{1}(3)+p_{2}(1)>p_{1}(3)+p_{2}(3)$.

Allocation $x^{4}$ is not Walrasian. If it was, then the following (in)equalities would hold:

(1) $p_{1}(1)+p_{2}(1)=p_{1}(3)+p_{2}(3), \quad(2) p_{1}(2)+p_{2}(2)=p_{1}(1)+p_{2}(2)$,

(3) $p_{1}(3)+p_{2}(3)=p_{1}(2)+p_{2}(1), \quad$ (4) $p_{1}(1)+p_{2}(2)>p_{1}(1)+p_{2}(1)$,

(5) $p_{1}(1)+p_{2}(3)>p_{1}(2)+p_{2}(2), \quad(6) p_{1}(3)+p_{2}(1)>p_{1}(3)+p_{2}(3)$.

By $(2)$ and (5), we obtain $p_{1}(1)+p_{2}(3)>p_{1}(1)+p_{2}(2)$. Thus, $p_{2}(3)>p_{2}(2)$. By $(4), p_{2}(2)>$ $p_{2}(1)$ and by $(6), p_{2}(1)>p_{2}(3)$. Hence, $p_{2}(3)>p_{2}(2)>p_{2}(1)>p_{2}(3)$; a contradiction.

Hence, $W(R)=\left\{x^{3}\right\}$. Furthermore, $R C(R)=S R C(R)=$ Core $(R)=\left\{x^{2}, x^{3}, x^{4}\right\}$.

To compute the limit invariant distribution, we proceed as indicated in Section 5.2.

Construction of Least Resistance Trees: By Theorem 2, $\gamma\left(x^{2}\right)=\gamma\left(x^{3}\right)=\gamma\left(x^{4}\right)=2$.

(a) $\mathcal{R C}(R)=\left\{\left\{x^{2}\right\},\left\{x^{3}\right\},\left\{x^{4}\right\}\right\}$. The following table lists the coalitions that facilitate a transition from an initial state (row) to a final state (column) with one mistake ${ }^{17}$ (we omit the diagonal elements as they are irrelevant for cycle-free graphs).

\begin{tabular}{|c|c|c|c|c|}
\hline & $x^{1}$ & $x^{2}$ & $x^{3}$ & $x^{4}$ \\
\hline$x^{1}$ & - & $\emptyset$ & $\emptyset$ & $\emptyset$ \\
\hline$x^{2}$ & $\{1\},\{2\},\{3\}$ & - & $\emptyset$ & $N$ \\
\hline$x^{3}$ & $\{1\},\{2\},\{3\}$ & $N$ & - & $N$ \\
\hline$x^{4}$ & $\{1\},\{2\},\{3\}$ & $N$ & $N$ & - \\
\hline
\end{tabular}

The asymmetry in this example is due to the fact that it takes two mistakes for a transition from $x^{2}$ to $x^{3}$ while all other transitions between core allocations need only one mistake (and $x^{1}$ can be accessed from any core allocation by one mistake of any singleton coalition).

\footnotetext{
${ }^{17}$ The agent who makes a mistake is uniquely determined in this example.
} 
(b) The following table lists the coalitions that facilitate a transition from an initial state (row) to a final state (column) with zero mistakes.

\begin{tabular}{|c|c|c|c|c|}
\hline & $x^{1}$ & $x^{2}$ & $x^{3}$ & $x^{4}$ \\
\hline$x^{1}$ & - & $N$ & $N$ & $N$ \\
\hline$x^{2}$ & $\emptyset$ & - & $\emptyset$ & $\emptyset$ \\
\hline$x^{3}$ & $\emptyset$ & $\emptyset$ & - & $\emptyset$ \\
\hline$x^{4}$ & $\emptyset$ & $\emptyset$ & $\emptyset$ & - \\
\hline
\end{tabular}

(c) There are $16 x^{2}$-trees. ${ }^{18}$

$$
\begin{aligned}
& \left\{\left[x^{3}, x^{2}\right],\left[x^{4}, x^{3}\right],\left[x^{1}, x^{2}\right]\right\} ;\left\{\left[x^{3}, x^{2}\right],\left[x^{4}, x^{3}\right],\left[x^{1}, x^{3}\right]\right\} ;\left\{\left[x^{3}, x^{2}\right],\left[x^{4}, x^{3}\right],\left[x^{1}, x^{4}\right]\right\} ; \\
& \left\{\left[x^{4}, x^{2}\right],\left[x^{3}, x^{4}\right],\left[x^{1}, x^{2}\right]\right\} ;\left\{\left[x^{4}, x^{2}\right],\left[x^{3}, x^{4}\right],\left[x^{1}, x^{3}\right]\right\} ;\left\{\left[x^{4}, x^{2}\right],\left[x^{3}, x^{4}\right],\left[x^{1}, x^{4}\right]\right\} ; \\
& \left\{\left[x^{3}, x^{2}\right],\left[x^{4}, x^{2}\right],\left[x^{1}, x^{2}\right]\right\} ;\left\{\left[x^{3}, x^{2}\right],\left[x^{4}, x^{2}\right],\left[x^{1}, x^{3}\right]\right\} ;\left\{\left[x^{3}, x^{2}\right],\left[x^{4}, x^{2}\right],\left[x^{1}, x^{4}\right]\right\} ; \\
& \left\{\left[x^{1}, x^{2}\right],\left[x^{4}, x^{3}\right],\left[x^{3}, x^{1}\right]\right\} ; \\
& \left\{\left[x^{1}, x^{3}\right],\left[x^{3}, x^{2}\right],\left[x^{4}, x^{1}\right]\right\} ; \\
& \left\{\left[x^{1}, x^{2}\right],\left[x^{3}, x^{4}\right],\left[x^{4}, x^{1}\right]\right\} ; \\
& \left\{\left[x^{1}, x^{4}\right],\left[x^{4}, x^{2}\right],\left[x^{3}, x^{1}\right]\right\} ; \\
& \left\{\left[x^{3}, x^{2}\right],\left[x^{1}, x^{2}\right],\left[x^{4}, x^{1}\right]\right\} ; \\
& \left\{\left[x^{4}, x^{2}\right],\left[x^{1}, x^{2}\right],\left[x^{3}, x^{1}\right]\right\} ; \\
& \left\{\left[x^{1}, x^{2}\right],\left[x^{4}, x^{1}\right],\left[x^{3}, x^{1}\right]\right\} ;
\end{aligned}
$$

There are $8 x^{3}$-trees.

$$
\begin{aligned}
& \left\{\left[x^{4}, x^{3}\right],\left[x^{2}, x^{4}\right],\left[x^{1}, x^{2}\right]\right\} ;\left\{\left[x^{4}, x^{3}\right],\left[x^{2}, x^{4}\right],\left[x^{1}, x^{3}\right]\right\} ;\left\{\left[x^{4}, x^{3}\right],\left[x^{2}, x^{4}\right],\left[x^{1}, x^{4}\right]\right\} ; \\
& \left\{\left[x^{4}, x^{2}\right],\left[x^{2}, x^{1}\right],\left[x^{1}, x^{3}\right]\right\} \\
& \left\{\left[x^{2}, x^{4}\right],\left[x^{4}, x^{1}\right],\left[x^{1}, x^{3}\right]\right\} ; \\
& \left\{\left[x^{2}, x^{1}\right],\left[x^{1}, x^{4}\right],\left[x^{4}, x^{3}\right]\right\} ; \\
& \left\{\left[x^{4}, x^{3}\right],\left[x^{2}, x^{1}\right],\left[x^{1}, x^{3}\right]\right\} ; \\
& \left\{\left[x^{2}, x^{1}\right],\left[x^{4}, x^{1}\right],\left[x^{1}, x^{3}\right]\right\}
\end{aligned}
$$

and $12 x^{4}$-trees.

$$
\begin{aligned}
& \left\{\left[x^{3}, x^{2}\right],\left[x^{2}, x^{4}\right],\left[x^{1}, x^{2}\right]\right\} ;\left\{\left[x^{3}, x^{2}\right],\left[x^{2}, x^{4}\right],\left[x^{1}, x^{3}\right]\right\} ;\left\{\left[x^{3}, x^{2}\right],\left[x^{2}, x^{4}\right],\left[x^{1}, x^{4}\right]\right\} ; \\
& \left\{\left[x^{2}, x^{4}\right],\left[x^{3}, x^{4}\right],\left[x^{1}, x^{2}\right]\right\} ;\left\{\left[x^{2}, x^{4}\right],\left[x^{3}, x^{4}\right],\left[x^{1}, x^{3}\right]\right\} ;\left\{\left[x^{2}, x^{4}\right],\left[x^{3}, x^{4}\right],\left[x^{1}, x^{4}\right]\right\} ; \\
& \left\{\left[x^{2}, x^{1}\right],\left[x^{1}, x^{3}\right],\left[x^{3}, x^{4}\right]\right\} ; \\
& \left\{\left[x^{1}, x^{4}\right],\left[x^{3}, x^{2}\right],\left[x^{2}, x^{1}\right]\right\} ; \\
& \left\{\left[x^{1}, x^{2}\right],\left[x^{2}, x^{4}\right],\left[x^{3}, x^{1}\right]\right\} ; \\
& \left\{\left[x^{3}, x^{4}\right],\left[x^{1}, x^{4}\right],\left[x^{2}, x^{1}\right]\right\} ; \\
& \left\{\left[x^{2}, x^{4}\right],\left[x^{1}, x^{4}\right],\left[x^{3}, x^{1}\right]\right\} ; \\
& \left\{\left[x^{1}, x^{4}\right],\left[x^{2}, x^{1}\right],\left[x^{3}, x^{1}\right]\right\} ;
\end{aligned}
$$

\footnotetext{
${ }^{18}$ Trees listed in one row only differ through a different connection of $x^{1}$. The first three rows depict the trees with direct edges between core allocations and all possible connections of $x^{1}$. The other trees are then constructed through all feasible paths from one core allocation to another via $x^{1}$.
} 
Computation of the Limit Invariant Distribution: By Assumption 4, the probability of a mistake does not depend on the allocation or the identity of the agent. Now suppose that the probability for a certain coalition to become active does also not depend on the allocation. Then, $\widetilde{m}\left(x^{2}, x^{1}\right)=\widetilde{m}\left(x^{3}, x^{1}\right)=\widetilde{m}\left(x^{4}, x^{1}\right)$. Moreover, assume that the grand coalition agrees upon recontracting on each core allocation with the same probability if the p.d.r. process is at $x^{1}$ (i.e., $\widetilde{m}\left(x^{1}, x^{2}\right)=\widetilde{m}\left(x^{1}, x^{3}\right)=\widetilde{m}\left(x^{1}, x^{4}\right)$ ). Finally, suppose that all transitions between core allocations that only need one mistake have the same probability (i.e., $\widetilde{m}\left(x^{2}, x^{4}\right)=$ $\left.\widetilde{m}\left(x^{3}, x^{2}\right)=\widetilde{m}\left(x^{3}, x^{4}\right)=\widetilde{m}\left(x^{4}, x^{2}\right)=\widetilde{m}\left(x^{4}, x^{3}\right)\right)$. Then Theorem 3 yields

$$
\begin{aligned}
& \mu^{*}\left(x^{2}\right)=9 \widetilde{m}\left(x^{1}, x^{2}\right)\left(\widetilde{m}\left(x^{2}, x^{4}\right)\right)^{2}+6 \widetilde{m}\left(x^{2}, x^{1}\right) \widetilde{m}\left(x^{1}, x^{2}\right) \widetilde{m}\left(x^{2}, x^{4}\right)+\widetilde{m}\left(x^{1}, x^{2}\right)\left(\widetilde{m}\left(x^{2}, x^{1}\right)\right)^{2} \\
& \mu^{*}\left(x^{3}\right)=3 \widetilde{m}\left(x^{1}, x^{2}\right)\left(\widetilde{m}\left(x^{2}, x^{4}\right)\right)^{2}+4 \widetilde{m}\left(x^{2}, x^{1}\right) \widetilde{m}\left(x^{1}, x^{2}\right) \widetilde{m}\left(x^{2}, x^{4}\right)+\widetilde{m}\left(x^{1}, x^{2}\right)\left(\widetilde{m}\left(x^{2}, x^{1}\right)\right)^{2} \\
& \mu^{*}\left(x^{4}\right)=6 \widetilde{m}\left(x^{1}, x^{2}\right)\left(\widetilde{m}\left(x^{2}, x^{4}\right)\right)^{2}+5 \widetilde{m}\left(x^{2}, x^{1}\right) \widetilde{m}\left(x^{1}, x^{2}\right) \widetilde{m}\left(x^{2}, x^{4}\right)+\widetilde{m}\left(x^{1}, x^{2}\right)\left(M\left(x^{2}, x^{1}\right)\right)^{2}
\end{aligned}
$$

which implies that $\mu^{*}\left(x^{2}\right)>\mu^{*}\left(x^{4}\right)>\mu^{*}\left(x^{3}\right)$. Note in particular that the unique Walrasian allocation $x^{3}$ is the worst predictor for the long-run behavior of the process.

\section{Example 4: Multiple Walrasian Allocations Recall}

$R_{1}:(1,2),(3,3),(2,3),(1,1)$, anything,

$R_{2}:(1,3),(1,2),(3,3),(2,2)$, anything,

$R_{3}:(2,1),(3,1),(3,3)$, anything,

and $\operatorname{IR}(R)=\left\{x^{1}, x^{2}, x^{3}, x^{4}\right\}$ with,

$$
\begin{aligned}
& x^{1}=\{(1,1),(2,2),(3,3)\}, \quad x^{2}=\{(2,3),(1,2),(3,1)\}, \quad x^{3}=\{(3,3),(1,2),(2,1)\}, \\
& x^{4}=\{(1,2),(3,3),(2,1)\} .
\end{aligned}
$$

Clearly, $x^{2}\{1,2,3\}$-blocks $x^{1}$ and $x^{3}\{1,2,3\}$-blocks $x^{2}$. Since $x^{3}$ and $x^{4}$ cannot be blocked by any coalition, $\operatorname{Core}(R)=\left\{x^{3}, x^{4}\right\}$. Next, we check if any of the core allocations is Walrasian.

Allocation $x^{3}$ is Walrasian. It is easy to check that the following (in)equalities hold, e.g., for price system $p \equiv\left(p_{1}, p_{2}\right)$ such that $p_{1}=(2,2,0)$ and $p_{2}=(1,2,3)$ :

(1) $p_{1}(1)+p_{2}(1)=p_{1}(3)+p_{2}(3), \quad(2) p_{1}(2)+p_{2}(2)=p_{1}(1)+p_{2}(2)$,

(3) $p_{1}(3)+p_{2}(3)=p_{1}(2)+p_{2}(1), \quad$ (4) $p_{1}(1)+p_{2}(2)>p_{1}(1)+p_{2}(1)$,

(5) $p_{1}(1)+p_{2}(3)>p_{1}(2)+p_{2}(2)$.

Allocation $x^{4}$ is Walrasian. It is easy to check that the following (in)equalities hold, e.g., for price system $p \equiv\left(p_{1}, p_{2}\right)$ such that $p_{1}=(2,1,2)$ and $p_{2}=(1,1,1)$ :
(1) $p_{1}(1)+p_{2}(1)=p_{1}(3)+p_{2}(3)$,
(2) $p_{1}(2)+p_{2}(2)=p_{1}(1)+p_{2}(2)$,
(3) $p_{1}(3)+p_{2}(3)=p_{1}(2)+p_{2}(1)$,
(4) $p_{1}(1)+p_{2}(2)>p_{1}(2)+p_{2}(2)$,
(5) $p_{1}(1)+p_{2}(3)>p_{1}(2)+p_{2}(2)$. 
Hence, $W(R)=\left\{x^{3}, x^{4}\right\}$.

By Theorem 1(iii) only individually rational allocations can be recurrent allocations. By Theorem 1(ii), $\left\{x^{3}\right\}$ and $\left\{x^{4}\right\}$ are recurrent classes. Since $x^{3}\{1,2,3\}$-blocks $x^{2}$ and $x^{4}\{1,2,3\}$-blocks $x^{1},\left\{x^{3}\right\}$ and $\left\{x^{4}\right\}$ are the only recurrent classes. Therefore, $R C(R)=$ $\operatorname{SRC}(R)=\operatorname{Core}(R)=\left\{x^{3}, x^{4}\right\}$.

\section{Example 5: No Walrasian Allocation and a Multi-Valued Core Recall}

$R_{1}:(1,2),(3,3),(2,3),(1,1)$, anything,

$R_{2}:(3,2),(1,2),(3,3),(2,2)$, anything,

$R_{3}:(1,3),(3,1),(2,3),(2,1),(3,3)$, anything,

and $\operatorname{IR}(R)=\left\{x^{1}, x^{2}, x^{3}, x^{4}, x^{5}\right\}$ with

$$
\begin{aligned}
& x^{1}=\{(1,1),(2,2),(3,3)\}, \quad x^{2}=\{(1,1),(3,2),(2,3)\}, \quad x^{3}=\{(2,3),(1,2),(3,1)\}, \\
& x^{4}=\{(3,3),(1,2),(2,1)\}, \quad x^{5}=\{(1,2),(3,3),(2,1)\} .
\end{aligned}
$$

Clearly, $x^{2}\{2,3\}$-blocks $x^{1}, x^{2}\{2,3\}$-blocks $x^{4}$, and $x^{2}\{2,3\}$-blocks $x^{5}$. Since $x^{2}$ and $x^{3}$ cannot be blocked by any coalition, $\operatorname{Core}(R)=\left\{x^{2}, x^{3}\right\}$. Next, we check if any of the core allocations is Walrasian.

Allocation $x^{2}$ is not Walrasian. If it was, then the following (in)equalities would hold:

(1) $p_{1}(2)+p_{2}(2)=p_{1}(3)+p_{2}(2), \quad(2) p_{1}(3)+p_{2}(3)=p_{1}(2)+p_{2}(3)$,

(3) $p_{1}(2)+p_{2}(3)>p_{1}(1)+p_{2}(1), \quad(4) p_{1}(3)+p_{2}(3)>p_{1}(1)+p_{2}(1)$

(5) $p_{1}(1)+p_{2}(2)>p_{1}(1)+p_{2}(1), \quad(6) p_{1}(3)+p_{2}(1)>p_{1}(3)+p_{2}(3)$,

(7) $p_{1}(1)+p_{2}(3)>p_{1}(3)+p_{2}(3)$.

By (5), $p_{2}(2)>p_{2}(1)$ and by (6), $p_{2}(1)>p_{2}(3)$. Hence, (8) $p_{2}(2)>p_{2}(3)$.

Next, by $(1), p_{1}(2)=p_{1}(3)$ and therefore, $p_{1}(3)+p_{2}(3)=p_{1}(2)+p_{2}$. By $(7), p_{1}(1)>p_{1}(3)$ and therefore, $p_{1}(1)+p_{2}(1)>p_{1}(3)+p_{2}(1)$. Using the previous (in)equalities, (3) implies $p_{1}(3)+p_{2}(3)>p_{1}(3)+p_{2}(1)$. Hence, $p_{2}(3)>p_{2}(1)$; a contradiction to $(8)$.

Allocation $x^{3}$ is not Walrasian. If it was, then the following (in)equalities would hold:

(1) $p_{1}(1)+p_{2}(1)=p_{1}(2)+p_{2}(3), \quad(2) p_{1}(2)+p_{2}(2)=p_{1}(1)+p_{2}(2)$,

(3) $p_{1}(3)+p_{2}(3)=p_{1}(3)+p_{2}(1), \quad$ (4) $p_{1}(3)+p_{2}(3)>p_{1}(1)+p_{2}(1)$,

(5) $p_{1}(1)+p_{2}(2)>p_{1}(1)+p_{2}(1), \quad(6) p_{1}(3)+p_{2}(2)>p_{1}(2)+p_{2}(2)$,

(7) $p_{1}(1)+p_{2}(3)>p_{1}(3)+p_{2}(3)$.

By (7), $p_{1}(1)>p_{1}(3)$ and by $(6), p_{1}(3)>p_{1}(2)$. By $(2), p_{1}(2)=p_{1}(1)$. Hence, $p_{1}(1)>$ $p_{1}(3)>p_{1}(2)=p_{1}(1)$; a contradiction.

Hence, $W(R)=\emptyset$.

By Theorem 1(iii) only individually rational allocations can be recurrent allocations. According to Theorem 1(ii) $\left\{x^{2}\right\}$ and $\left\{x^{3}\right\}$ are (singleton) recurrent classes. Since $x^{2}\{1,2,3\}$ block $x^{1}$ and $x^{2}\{2,3\}$-blocks $x^{4}$ and $x^{5},\left\{x^{2}\right\}$ and $\left\{x^{3}\right\}$ are the only recurrent classes. Therefore, $R C(R)=S R C(R)=\operatorname{Core}(R)=\left\{x^{2}, x^{3}\right\}$. 


\section{B A Short Markov Process Dictionary}

- A Markov process $(X, M)$ is determined by a discrete state space $X$ and a mapping $M: X \times X \rightarrow[0,1]$ where $M\left(x, x^{\prime}\right)$ describes the probability that the state equals $x^{\prime} \in X$ in period $t+1$ whenever it was in $x \in X$ in period $t$. Clearly, $\sum_{x^{\prime} \in X} M\left(x, x^{\prime}\right)=1$. Here we restrict ourselves to finite, time-homogeneous Markov processes, i.e., $X$ is a finite set and transition probabilities induced (and captured in $M$ ) do not depend on time.

- A recurrent class $A \subseteq X$ is a minimal set of allocations that once entered throughout the dynamic process is never abandoned, i.e., for all $x \in A$ and $x^{\prime} \notin A, M\left(x, x^{\prime}\right)=0$. Allocations that do not belong to any recurrent class are called transient.

- A recurrent class is aperiodic whenever it does not contain any deterministic and nontrivial cycle, i.e., there is no sequence of at least two allocations $\left\{x_{1}, x_{2}, \ldots, x_{n}\right\}$ such that for all $i=1, . ., n-1, M\left(x_{i}, x_{i+1}\right)=M\left(x_{n}, x_{1}\right)=1$. Note that a sufficient condition for the aperiodicity of a recurrent class $A$ is that there is an $x \in A$ such that $M(x, x)>0$, i.e., that the Markov process exhibits sufficient inertia.

- Every Markov process induces (a set of) invariant distributions $\mu: X \rightarrow[0,1]$ with $\sum_{x \in X} \mu(x)=1$ and $\mu \cdot M=\mu$. Every recurrent class $A \subseteq X$ corresponds to exactly one invariant distribution with support $A$, i.e., $\sum_{x \in A} \mu(x)=1$. The set of all invariant distributions of a Markov process is the convex hull of the invariant distributions of all its recurrent classes. The support of an invariant distribution $\mu$ is therefore a (non-empty) set of recurrent classes.

- A Markov process is ergodic if it has a unique recurrent class. The invariant distribution of an ergodic Markov process is unique.

- A Markov process is called irreducible if it is ergodic and the unique recurrent class coincides with the state space $X$.

- By the fundamental theorem of Markov processes an invariant distribution which is induced by an aperiodic recurrent class $A \subseteq X$ describes the probability that the process will be at allocation $x$ if it reached an allocation in $A$ and propagated forever, i.e., for all $x \in A$ and all probability distributions over allocations $\nu: X \rightarrow[0,1]$ whose support is contained in $A, \mu(x)=\lim _{T \rightarrow \infty} \nu \cdot P^{T}$.

- A perturbed Markov process $\left(X, M^{\epsilon}\right)$ is a Markov process such that all transition probabilities $M^{\epsilon}\left(x, x^{\prime}\right)$ are continuous in $\epsilon$, and for all $x, x^{\prime} \in X, \lim _{\epsilon \rightarrow 0} M^{\epsilon}\left(x, x^{\prime}\right)=M\left(x, x^{\prime}\right)$. More specifically, $M^{\epsilon}\left(x, x^{\prime}\right)>0$ for $\epsilon>0$ implies that there is an $r \geq 0$ such that $\infty>\lim _{\epsilon \rightarrow 0} \epsilon^{-r} M^{\epsilon}\left(x, x^{\prime}\right)>0$. Hence, we restrict ourselves to regular perturbations and $M^{\epsilon}$ is polynomial in $\epsilon$, i.e., we can write $M^{\epsilon}\left(x, x^{\prime}\right)=\sum_{i=0, \ldots, \hat{\imath}} m_{i}\left(x, x^{\prime}\right) \epsilon^{i}$ where $\hat{\imath}$ is a finite number and $m\left(x, x^{\prime}\right)>0$ for at least some $i$.

- The limit invariant distribution $\mu^{*}$ of a Markov process $(X, M)$ is the invariant distribution $\mu^{\epsilon}$ of a perturbed process $\left(X, M^{\epsilon}\right)$ in the limit of $\epsilon \rightarrow 0$. Note that any perturbed Markov process is irreducible, hence its invariant distribution is unique. Moreover, $\lim _{\epsilon \rightarrow 0} \mu^{\epsilon} \equiv \mu^{*}$ exists and is an invariant distribution of $(X, M)$.

- An allocation in the support of $\mu^{*}$ is stochastically stable. 


\section{References}

Feldman, A. (1974): "Recontracting Stability." Econometrica, 42: 35-44.

Freidlin, M. and Wentzell, A. (1998): Random Perturbations of Dynamical Systems. Springer Verlag, New York.

Green, J. (1974): "The Stability of Edgeworth's Recontracting Process." Econometrica, 42: $21-34$.

Jackson, M. and Watts, A. (2002): "The Evolution of Social and Economic Networks." Journal of Economic Theory, 106: 265-295.

Kandori, M., Mailath, G. J., and Rob, R. (1993): "Learning, Mutation, and Long-Run Equilibria in Games." Econometrica, 61: 29-56.

Klaus, B. (2006): "The Coordinate-Wise Core for Multiple-Type Housing Markets is SecondBest Incentive Compatible." METEOR Working Paper RM/05/019.

Konishi, H., Quint, T., and Wako, J. (2001): "On the Shapley-Scarf Market: the Case of Multiple Indivisible Goods." Journal of Mathematical Economics, 35: 1-15.

Moulin, H. (1995): Cooperative Microeconomics: A Game Theoretic Introduction. Princeton University Press, Princeton, NJ.

Roth, A. E. and Postlewaite, A. (1977): "Weak versus Strong Domination in a Market with Indivisible Goods." Journal of Mathematical Economics, 4: 131-137.

Serrano, R. and Volij, O. (2005): "Mistakes in Cooperation: the Stochastic Stability of Edgeworth's Recontracting." Working Paper.

Shapley, L. and Scarf, H. (1974): "On Cores and Indivisibility." Journal of Mathematical Economics, 1: 23-37.

Young, H. P. (1993): "The Evolution of Conventions." Econometrica, 61: 57-84. 